\title{
Epitaxial ferroelectric oxide thin films for optical applications
}

Cite as: Appl. Phys. Rev. 5, 041108 (2018); https://doi.org/10.1063/1.5046559

Submitted: 29 June 2018 . Accepted: 05 November 2018 . Published Online: 06 December 2018

D. Sando, Yurong Yang, Charles Paillard (D), B. Dkhil, L. Bellaiche, and V. Nagarajan

\section{COLLECTIONS}

F This paper was selected as Featured
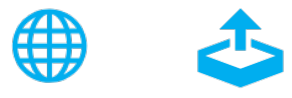

\section{ARTICLES YOU MAY BE INTERESTED IN}

Domain switching in single-phase multiferroics

Applied Physics Reviews 5, 021102 (2018); https://doi.org/10.1063/1.5018872

$\mathrm{BaTiO}_{3}$-based piezoelectrics: Fundamentals, current status, and perspectives Applied Physics Reviews 4, 041305 (2017); https://doi.org/10.1063/1.4990046

Atomic-scale mapping of interface reconstructions in multiferroic heterostructures Applied Physics Reviews 5, 041110 (2018); https://doi.org/10.1063/1.5053426

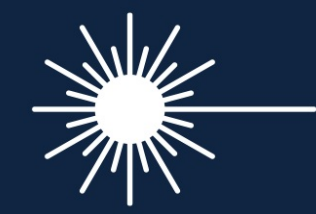

\section{AVS Quantum Science}

\section{SPECIAL ISSUE: Quantum Sensing and Metrology}




\title{
Epitaxial ferroelectric oxide thin films for optical applications
}

\author{
D. Sando, ${ }^{1, a)}$ Yurong Yang, ${ }^{2,3}$ Charles Paillard, ${ }^{3,4}$ B. Dkhil, ${ }^{4}$ L. Bellaiche, ${ }^{3}$ \\ and V. Nagarajan ${ }^{1, b)}$ \\ ${ }^{1}$ School of Materials Science and Engineering, UNSW Sydney, High Street, Kensington 2052, \\ New South Wales, Australia \\ ${ }^{2}$ National Laboratory of Solid State Microstructures, College of Engineering and Applied Sciences, \\ Nanjing University, Nanjing, China \\ ${ }^{3}$ Department of Physics and Institute for Nanoscience and Engineering, University of Arkansas, Fayetteville, \\ Arkansas 72701, USA \\ ${ }^{4}$ Laboratoire Structures, Propriétés et Modélisation des Solides, CentraleSupélec, CNRS-UMR8580, \\ Université Paris-Saclay, 91190 Gif-sur-Yvette, France
}

(Received 29 June 2018; accepted 5 November 2018; published online 6 December 2018)

Ferroelectrics are non-centrosymmetric crystalline materials that possess a spontaneous polarization that can be switched by an electric field. The electric-field-dependent optical response of these materials makes them important for optical devices, such as modulators or beam deflectors. In the inexorable drive to miniaturization, the concept of integrated thin film optical devices has led to the incorporation of ferroelectric thin films on single-crystal substrates. These structures have appealing electro-optic modulation characteristics, interesting strain-dependent bandgaps and refractive index, as well as promising possibilities for solar harvesting. Here, we review the work on epitaxial ferroelectric (FE) films for optical applications. We first show that FE thin film materials are attractive for integrated electro-optic modulators and then show that epitaxial strain can be used to enhance the FE and optical functionality of films. Next, we describe some of the photovoltaic functionality of FE thin film materials' systems and conclude the review by highlighting some thin-film devices that exploit the aforementioned optical effects. Published by AIP Publishing.

https://doi.org/10.1063/1.5046559

\section{TABLE OF CONTENTS}

I. INTRODUCTION

II. ELECTRO-OPTIC EFFECT IN THIN-FILM

FERROELECTRICS ....................

A. A brief history of thin film ferroelectric technologies for electro-optic devices ......

B. Understanding the role of thin film processing and its influence on device properties ........................

C. La-substituted lead zirconate titanate (PLZT) and related PZT compounds .......

D. Lithium niobate....................

E. Barium titanate and related compounds.....

F. Bismuth ferrite ......................

G. Other ferroelectric films for EO modulation

III. STRAIN MODULATION OF OPTICAL

PARAMETERS ......................

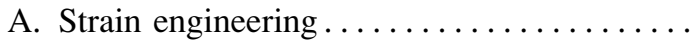

B. Theoretical approaches for strain modulated optical parameters

\footnotetext{
a)daniel.sando@unsw.edu.au

b)nagarajan@unsw.edu.au
}

C. Large elasto-optic effects in ferroelectric thin films .......................

D. Elasto-optic effects in ferroelectric oxide thin films $-\mathrm{PbTiO}_{3}$ as a test case $\ldots \ldots \ldots$.

E. Experimental reports on strain-modulated optical properties................... 12

F. Bandgap engineering............... 13

IV. BULK PHOTOVOLTAIC EFFECT IN THIN FILM FERROELECTRICS . . . . . . . . . . . . . 14

V. DEVICE PROTOTYPES .................. 17

A. Design rules for optical thin films for successful device integration............

B. Electro-optic guided-wave modulator device prototypes based on FE thin films ........ 17

C. Devices based on PLZT and related compounds ...................... 20

D. Barium titanate $\ldots \ldots \ldots \ldots \ldots \ldots \ldots \ldots, 20$

E. Bismuth ferrite ................... 20

VI. CONCLUSIONS ......................... 21

\section{INTRODUCTION}

The field of integrated optics is almost half a century old. Initially put forward by Miller in 1969 (Ref. 1), it proposed to use refractive index changes in glass substrates to create "laser beam circuitry." The ultimate goal and 
motivation was miniaturization, and economy, presumably in both fabrication costs and energy efficiency. A large portion of the demand for optoelectronic technologies came from the development of the so-called electro-optic modulator, which converts an electrical signal to an optical signal suitable for transport through optical fibers. Starting in the 1970s, there was a strong drive to use ferroelectric (FE) thin films to design integrated electro-optic (EO) modulators. Another attractive aspect of integrated optics was the possibility, particularly when using thin films, to interface directly with semiconductor lasers, and thus realize all-optical circuitry. $^{2}$

In this regard, complex ferroelectric oxide heterostructures have been widely explored for use as materials with attractive electro-optic properties. This is chiefly derived from the fact that their respective bulk parent compounds exhibit strong optical responses to external stimuli such as electric field or strain. The aim of the present review is to introduce the reader to the unique optical functionalities that complex ferroelectric oxide thin films offer, particularly with a view to integrated optics, ${ }^{3}$ photonics, ${ }^{4}$ and solar harvesting. ${ }^{5}$ While the materials requirements for EO applications (good transparency, refractive index between the film and the substrate, etc.) and photovoltaic applications (good absorption, appropriate work function between the film and electrodes) are very different, we show that using FE materials, depending strongly on the processing methods and device design, these seemingly opposing requirements can be overcome.

We have chosen to focus on epitaxial films since they are promising in integrated optics applications. The primary reasons are the lack of scattering losses from grain boundaries that can occur in polycrystalline films with grain sizes approaching the light wavelength, as well as the distribution of the optical axis direction which could be random and thus affect the optical anisotropy. That being said, it is important to highlight that if the grain size is considerably smaller than the operation wavelength, then polycrystalline films can offer promising avenues for optical devices (examples of which can be found in Refs. 6 and 7). The use of epitaxial films puts strict limitations on fabrication techniques, but high-quality thin films are routinely obtained in research laboratories. Large scale commercialization of chemical routes or physical vapor deposition techniques would encourage further development in this direction.

The motivation to use oxide ferroelectric thin films arises from the fact that for optical applications, traditionally such materials have been the most promising bulk versions of ferroelectrics. Another important aspect in this context is the versatility of oxides to be able to grow in thin films under rather large levels of misfit strain - by virtue of the capability of oxides to form misfit dislocations and to form new phases to accommodate strain. ${ }^{8}$

The paper begins by introducing the electro-optic effect, including the fundamental mathematical framework that helps us to quantify the observed field-induced electro-optical phenomena. We then cover the development of various candidate materials for EO thin films over the past three decades. This is followed by a discussion of the first-principles-based theoretical construct required to accurately predict and rationalize epitaxial strain-induced modulation of optical properties, such as bandgap and refractive index. Using this framework, we show that thin films can offer giant modifications of their refractive index when strain is applied. We then move to experimental efforts that capture the influence of epitaxial strain on the optical response of such heterostructures. The latter parts of the paper cover the concept of "bandgap engineering" of ferroelectrics, and how this influences the bulk photovoltaic response, thereby paving the way for photoferroelectric materials. We conclude by highlighting some promising optical/photonic devices for future applications and critical open questions.

\section{ELECTRO-OPTIC EFFECT IN THIN-FILM FERROELECTRICS}

We begin with the so-called "electro-optic effect": the change in refractive index of a medium upon the application of an electric field. Before going into the material's details, it is instructive to introduce the principle relationships that govern electro-optical behavior (for full details, the reader is referred to a standard text on the topic, e.g., Ref. 9). This phenomenon is widely used in the electro-optic modulator, an indispensable element in modern telecommunications. In this device, an electrical signal is converted to an optical signal, through the electro-optic effect. More formally, the EO effect is described by the change in refractive index $\Delta n(E)$ of a medium, through

$$
\Delta n(E)=-\frac{1}{2} r n^{3} E-\frac{1}{2} R n^{3} E^{2},
$$

where $n$ is the refractive index, $E$ is the applied electric field, and $r$ and $R$ are the linear (Pockels) and quadratic (Kerr) EO coefficients, respectively. The EO $r$ and $R$ quantities are third and fourth rank tensors, respectively; however, in the cases dealt with in this review, an effective coefficient suffices to describe the phenomenon. In FE crystals with a well-defined direction of polarization (and therefore optic axis), the linear effect dominates, and so often only the first term of the righthand side of Eq. (1) is used.

For traveling-wave EO phase modulators [Fig. 1(a)], the half wave voltage $V_{\pi}$ is an important metric. It defines, for a given modulator geometry and dimensions, the voltage required to induce a $\pi$ phase shift in the modulated beam, in a transverse configuration [Fig. 1(a)]

$$
V_{\pi}=\frac{\lambda d}{\left(L r_{33} n^{3}\right)} .
$$

Here, $\lambda$ is the wavelength, $d$ is the spacing between the electrodes, $L$ is the length of the modulator, $r_{33}$ is the EO coefficient, and $n$ is the refractive index. It follows from Eq. (2) that in order to obtain low-voltage operation, either large EO coefficients or long devices are required.

In thin films, the EO effect is most conveniently measured in a transmission geometry using planar electrodes, ${ }^{10,11}$ which usually allows only the determination of an effective EO coefficient. Other techniques include prism-coupling, ${ }^{12}$ 
(a)

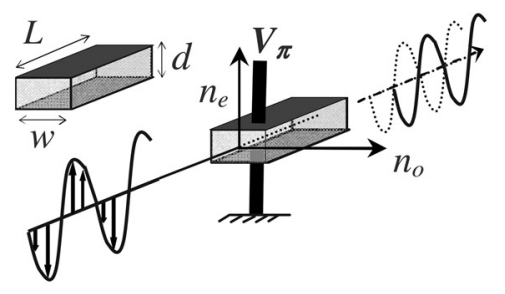

(b)

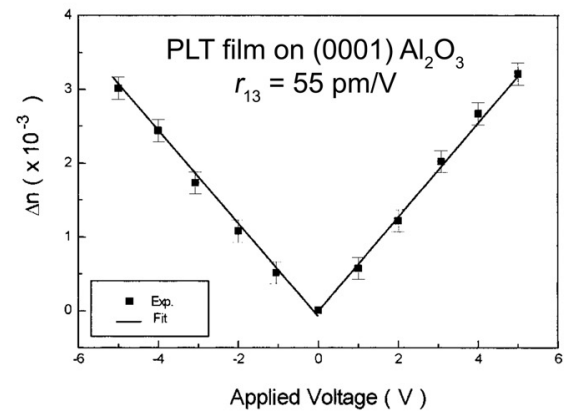

(c)

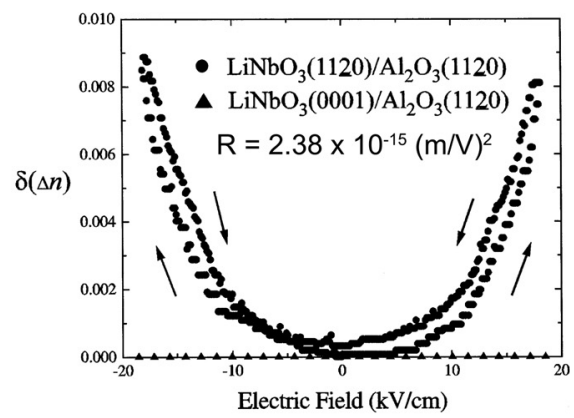

FIG. 1. (a) Schematic depicting the modulation of a light wave using the electro-optic effect. Reproduced with permission from J. Appl. Phys. 109, 014107 (2011). Copyright 2011 AIP Publishing LLC. ${ }^{253}$ (b) Example of the linear birefringence change in a PLZT thin film. Reproduced with permission from J. Appl. Phys. 85, 1780 (1999). Copyright 1999 AIP Publishing LLC. (c) Quadratic EO effect in $\mathrm{LiNbO}_{3}$ films of different orientations. Reproduced with permission from D. Kim et al., Mater. Sci. Eng. B 56, 251 (1998). Copyright 1998 Elsevier. $^{254}$

waveguide mode measurements, ${ }^{13}$ modified ellipsometry, ${ }^{14}$ or beam deflection. ${ }^{15}$ In order to exhibit the linear EO effect [Fig. 1(b)] and to enable extraction of different elements of the EO tensor (i.e., $r_{i j}$ coefficients), the FE film must have a single ferroelectric domain with a well-defined crystallographic orientation. ${ }^{16,17}$ This can be achieved by poling the FE material with electric field during growth, by applying a field while cooling through $T_{\mathrm{C}}$ (Refs. 18 and 19), or by applying a field larger than the coercive to pole the film. ${ }^{20}$ It is important to note that the success of forming single domain films using poling techniques will be highly dependent on the mechanical boundary conditions. In most cases, the existence of ferroelastic domains will mean that the polarization direction will not be changeable. Only in specific cases are "perfectly" single domain films possible by poling. Often when the film does not comprise a single ferroelectric domain, a quadratic EO effect is observed [see, for example, Fig. 1(c)].

The material of choice for macroscopic EO modulators is $\mathrm{LiNbO}_{3}$ (LNO), which crystallizes in a rhombohedral structure with the $R 3 c$ polar space group. LNO has been used for over 40 years in modulators ${ }^{21}$ and offers further functionalities such as holographic memory, ${ }^{22} 3 \mathrm{D}$ data storage, ${ }^{23}$ and encryption. ${ }^{24}$ Although it has modest EO coefficients (Table I), LNO can be grown by the Czochralski technique in large crystals (dimensions of $5 \mathrm{~cm}$ diameter and $10 \mathrm{~cm}$ length can routinely be achieved ${ }^{25}$ ) with the high optical quality required for devices. There are other ferroelectrics-such as $\mathrm{BaTiO}_{3}$ (BTO) -with larger EO coefficients, but the growth of large crystals is challenging; for example, the typical size of BTO crystals is $\sim 1 \mathrm{~cm}^{8}$

In the 1990s, there was a significant drive to integrate EO modulators on-chip, to reduce device footprint, to aid miniaturization, and to reduce power consumption. The key obstacles to this endeavor were mainly related to material and fabrication issues. Typically, in thin films, the EO coefficients do not approach the values that are possible in bulk compounds; this is due to the fact that thin films exhibit process-dependent properties such as differences in stoichiometry, the presence of defects, roughness, grain boundaries, strain gradients, and domain structures. ${ }^{15}$ It thus became clear that to realize reliable devices it was inevitable to thoroughly understand the structure-processing-property correlations for each material system. Section II A describes the materials development efforts for complex oxide ferroelectrics in electro-optic applications of the past three decades.

\section{A. A brief history of thin film ferroelectric technologies for electro-optic devices}

To prepare epitaxial films suitable for optical applications, various film growth techniques have been used. These

TABLE I. Ferroelectric characteristics (Curie transition temperature $T_{\mathrm{C}}$ and spontaneous polarization $P_{\mathrm{s}}$ ) and electro-optic performance [electro-optic coefficient $r$; refractive index $n$ at $633 \mathrm{~nm}$; dielectric constant $\varepsilon\left(\varepsilon_{a}\right.$ is orthogonal to polarization direction and $\varepsilon_{c}$ is along polarization direction) (clamped; i.e., constant strain, unless otherwise stated); and figure of merit $n^{3} r / \varepsilon_{a}$ ] for bulk ferroelectric oxides (this assumes use of $\varepsilon_{a}$, which is consistent with $a$-axis oriented traveling wave modulators). If temperature is not given, the values are assumed to be at room temperature.

\begin{tabular}{|c|c|c|c|c|c|c|c|c|}
\hline Material & $T_{\mathrm{C}}\left({ }^{\circ} \mathrm{C}\right)$ & $P_{\mathrm{s}}\left(\mu \mathrm{C} / \mathrm{cm}^{2}\right)\left(\right.$ at $\left.\mathrm{T}^{\circ} \mathrm{C}\right)$ & $r(\mathrm{pm} / \mathrm{V})$ & $n$ at $633 \mathrm{~nm}$ & $\varepsilon_{\mathrm{a}}\left(\right.$ at $\left.T^{\circ} \mathrm{C}\right)$ & $\varepsilon_{\mathrm{c}}\left(\right.$ at $\left.T^{\circ} \mathrm{C}\right)$ & $n^{3} r / \varepsilon(\mathrm{pm} / \mathrm{V})$ & References \\
\hline $\mathrm{LiNbO}_{3}$ & 1195 & $71(23)$ & $r_{33}=31.8$ & 2.29 & 84 & 30 & 12.6 & $26-28$ \\
\hline $\mathrm{BaTiO}_{3}$ & 120 & $26(23)$ & $r_{42}=1640$ & 2.37 & $\sim 4500(23)$ & $\sim 160(23)$ & 5.0 & 27 and 28 \\
\hline $\mathrm{LiTaO}_{3}$ & 610 & 50 & $r_{33}=31$ & 2.23 & 51 & 45 & 8.0 & 26 and 27 \\
\hline $\mathrm{PbTiO}_{3}$ & 490 & 66 & $r_{33}=5.9$ & 2.67 & 115 & 51 & 2.2 & 29-31 \\
\hline $\mathrm{KNbO}_{3}$ & 435 & $30(250)$ & $r_{33}=64$ & 2.23 & 160 (free) & 55 (free) & 13.0 & 28,32 , and 33 \\
\hline $\mathrm{K}\left(\mathrm{Ta}_{0.54} \mathrm{Nb}_{0.46}\right) \mathrm{O}_{3}{ }^{\mathrm{a}}$ & 50 & & $r_{\text {eff }}=2000$ & 2.35 & $\sim 2000$ (23) & & 96.1 & 19 and 34 \\
\hline $\mathrm{BiFeO}_{3}$ & 1100 & $\sim 100$ & $r_{\text {eff }}=12($ film $)$ & 3.0 & $30-50$ & $30-50$ & 8.1 & 20,35 , and 36 \\
\hline
\end{tabular}

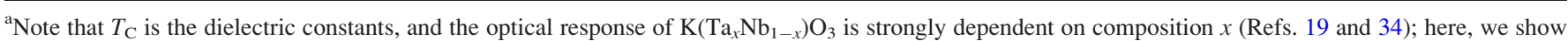
data for $x=0.54$. Table I is adapted from Ref. 37 . 
include chemical solution deposition, metal-organic chemical vapor deposition (MOCVD), sputtering, oxide molecular beam epitaxy (MBE), and pulsed laser deposition (PLD). It is not the intention of this review to describe these techniques; the reader is directed to various resources on such topics. ${ }^{38}$ Particularly, in the late 1980 s and early 1990s, leaps and bounds were made in oxide heteroepitaxy. Techniques such as MBE and PLD became commonplace and enabled epitaxial films with crystalline quality approaching that of single crystals grown by conventional solid-state routes.

Early reports on the synthesis and characterization of thin film ferroelectrics for electro-optics predominantly deal with PLZT (lanthanum-substituted lead zirconate titanate; $\left.\mathrm{La}-\mathrm{PbZr}_{\mathrm{x}} \mathrm{Ti}_{1-\mathrm{x}} \mathrm{O}_{3}\right)$. This material was chosen based on its attractive optical properties in ceramic form. ${ }^{39}$ On the other hand, the rapid strides in oxide thin film technologies in the 1990s finally allowed the exploration of the growth of epitaxial LNO thin films. Previously, bulk LNO was the "gold standard" for EO modulation, and it was hoped that such properties would translate to the thin film regime. However, the epitaxial growth of LNO is fraught with complications. ${ }^{40}$ This is further compounded by the sensitivity of LNO performance to the film's domain structure and surface roughness. As a result, optical losses were inevitable in waveguide architectures, and, more importantly, the EO coefficients were not close to those available in the bulk counterpart. These challenges notwithstanding, and there are various reports of successful growth and characterization of the optical properties of LNO, as discussed in a recent review. ${ }^{41}$ At the same time, barium titanate $\left(\mathrm{BaTiO}_{3}-\mathrm{BTO}\right)$ emerged as a promising alternative by virtue of its large EO coefficients (Table I). This spurred intense efforts into development of epitaxial BTO thin films by MOCVD, PLD, and MBE. ${ }^{3}$ Rapid developments in the improvement of film quality, and furthermore, the integration of such oxide films on $\mathrm{Si}^{42}$ shifted the focus to BTO and other related ferroelectrics with the simple $\mathrm{ABO}_{3}$ perovskite structure. Before discussing the EO response of various candidate $\mathrm{FE}$ oxide films, in Sec. II B, we discuss in more detail some of the complications regarding optical losses and the typically required structural characterizations needed to obtain optical thin films of sufficient quality for optical applications.

\section{B. Understanding the role of thin film processing and its influence on device properties}

A critical issue with EO films is optical losses in thin film waveguide structures. ${ }^{43}$ From a material's perspective, the main causes for optical loss in epitaxial structures are (i) scattering from domain or twin boundaries where the birefringence of the material changes; (ii) scattering losses associated with surface and interfacial roughness (see below); and (iii) defect states in the gap. The dependence of scattering loss on surface roughness can be described for waveguide structures as ${ }^{44}$

$$
\alpha_{s c}=\left(\frac{4 \pi \sigma}{\lambda}\right)^{2} \frac{f(\theta)}{t}
$$

where $\sigma$ is the root mean square (rms) surface roughness, $\lambda$ is the light wavelength, $f(\theta)$ is a geometric parameter related to the angle of reflection, and $t$ is the film thickness. It follows from Eq. (3) that thicker films and longer wavelengths result in lower scattering losses, while an increase in the surface roughness induces larger losses.

Optical losses were discussed extensively by Fork et al. in 1995 (Ref. 45). Detailed calculations showed at that time that a benchmark level of losses for waveguides at $1 \mathrm{~dB} / \mathrm{cm}$. To achieve this, upper limits of the morphological roughness of the surface is the main limitation. It was suggested later that, however, optical losses of $\sim 2 \mathrm{~dB} / \mathrm{cm}$ would be acceptable $^{37}$ and therefore roughness values of up to 2-4 nm would fall within such limitations. An important point regarding large optical losses is that often the defects responsible for losses are at such low densities that they may not be detectable in standard $\mathrm{x}$-ray diffraction scans. ${ }^{46}$

The above discussion leads to a key conclusion: fabrication of high-quality films is paramount for optical applications. Figure 2 summarizes the most important characterizations typically carried out to assess stoichiometry, structure, and surface morphology of films before optical characterization. As a first step, x-ray diffraction (XRD) is typically used to confirm the phase purity of the film $[\theta-2 \theta$ scan; Fig. 2(a)] and the epitaxial relationship between the film and the substrate $[\varphi$-scan, Fig. 2(b)]. This latter step also allows to determine the existence of rotational twins or similar. ${ }^{47}$ It is also appropriate to confirm that the films exhibit reliable ferroelectricity $[P-E$ hysteresis loop, Fig. 2(c)], often in a capacitor structure (patterned top electrode of a metal, lower electrode of some conductive oxide such as $\mathrm{SrRuO}_{3}$ ). Since scattering loss is a serious issue for optical devices, it is important to measure the roughness of the surface, usually by atomic force microscopy scans [Fig. 2(d)]. Relating to the same aspect, transmission electron microscopy (TEM) is useful for checking the interface (substrate-film) quality [Fig. 2(e)], as roughness here will also induce scattering losses. Finally, the optical bandgap can be estimated from absorption data taken from transmittance or ellipsometry measurements, using a so-called Tauc plot [Fig. 2(f)], as first described in Ref. 48. Having described the requirements for film quality and structural and surface properties, we now move to a discussion of each material system from the perspective of the EO response.

\section{La-substituted lead zirconate titanate (PLZT) and related PZT compounds}

Research into the EO effect for ferroelectric thin films began in the 1970s with PLZT, La-substituted $\mathrm{PbZr}_{\mathrm{x}} \mathrm{Ti}_{1-\mathrm{x}} \mathrm{O}_{3}$ (PZT). PZT is a well-known high-performance piezoelectric, ${ }^{49}$ and the addition of lanthanum improves optical transparency. ${ }^{39}$ There are numerous good reviews ${ }^{18,39,50}$ on the topic of PLZT thin films grown by various methods; we do not repeat that work here, and we simply wish to highlight some important points. In the following, we refer to PLZT compositions using the nomenclature PLZT $(x / y / 1-y)$ denoting $\left(\mathrm{Pb}_{1-x} \mathrm{La}_{x}\right)\left(\mathrm{Zr}_{y} \mathrm{Ti}_{1-y}\right) \mathrm{O}_{3}$.

PLZT films have been grown by sol-gel, sputtering, ${ }^{10,14,18}$ etc., mostly on sapphire $\left(\mathrm{Al}_{2} \mathrm{O}_{3}\right),{ }^{18,51} \mathrm{SrTiO}_{3}$ 
(a)

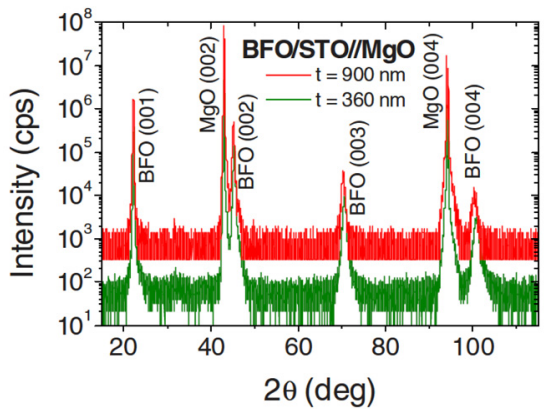

(d)

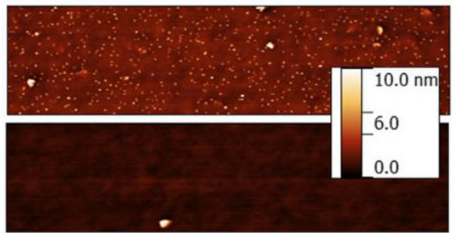

(b)

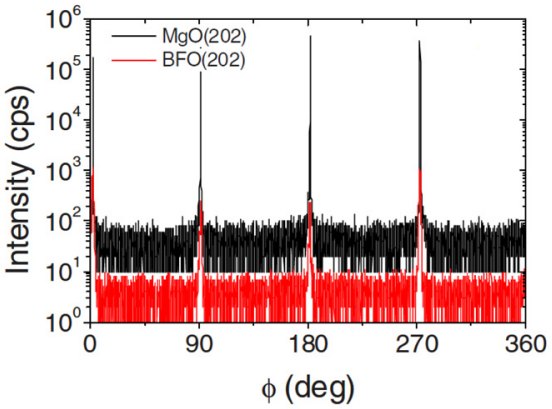

(e)

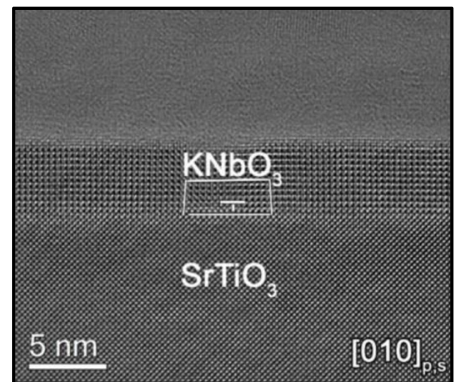

(c)

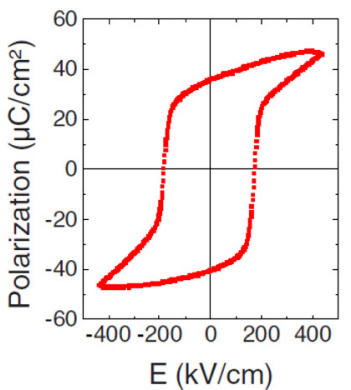

(f)

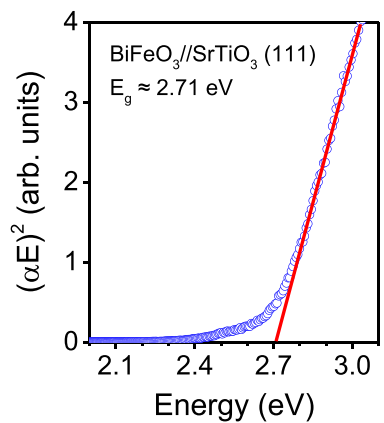

FIG. 2. Standard structural, ferroelectric, and optical characterizations for epitaxial films. (a) $\theta$-2 $\theta$ scan to confirm phase purity; (b) $\Phi$-scan to confirm epitaxial relationship between the film and the substrate (in this case, cube-on-cube); (c) P-E hysteresis loop to demonstrate ferroelectricity; (d) AFM topography scans $(4 \mu \mathrm{m} \times 2 \mu \mathrm{m})$ of BTO film on $\mathrm{Si}$, to confirm low surface roughness: top image shows particles of $\mathrm{BaO}_{\mathrm{x}}$, bottom shows film after soaking in water for $4 \mathrm{~h}$; the particles are removed (adapted from Ref. 255); (e) TEM to inspect crystallinity, interface roughness, and domain structure (adapted from Ref. 256); (f) Tauc plot to estimate optical bandgap, here shown for a $\mathrm{BiFeO}_{3}$ film growth epitaxially on $\mathrm{SrTiO}_{3}$ (111) substrate. (a)-(c) Reproduced with permission from Appl. Phys. Lett. 96, 182902 (2010). Copyright 2010 AIP Publishing LLC. (d) Reproduced with permission from K. J. Kormondy et al., Microelectron. Eng. 147, 215 (2015). Copyright 2015 Elsevier. (e) Reproduced with permission from M. Tyunina et al., New J. Phys. 17, 043048 (2015). Copyright 2015 IOP Publishing.

(STO) (Ref. 52), Nb-doped STO (Nb:STO) (Refs. 14 and 53), and some cases on $\mathrm{Si}$, with appropriate buffer layers. ${ }^{54}$ The composition of PLZT has a strong effect on the structural and ferroelectric properties, as well as the existence of "mixed phases," cf. the phase diagram of Fig. 3(a). For thin tetragonal-like films, the La content affects ferroelectricity by (1) reducing the tetragonality (8\% La gives a "pseudocubic" structure $)^{55}$ and (2) reducing the Curie temperature from $500{ }^{\circ} \mathrm{C}$ at $0 \%$ La to $140{ }^{\circ} \mathrm{C}$ with $28 \% \mathrm{La}^{17}$ The ferroelectricity is destroyed for compositions of La above $28 \% .^{56}$

Interestingly, it has been found that removing the $\mathrm{Zr}$ from the composition, i.e., PLT, can improve optical transparency. ${ }^{39,56}$ For instance, Okuyama et al. ${ }^{57}$ found the highest transparency at a composition of (14/0/100), i.e., 14\% La, and no Zr. While the exact mechanism for such a dependence on the $\mathrm{Zr}$ composition was not explained in Refs. 56 and 57, looking at the phase diagram of Fig. 3(a) may shed some light. The composition (14/0/100) appears to be well into the FE tetragonal region of the phase diagram, and it is thus well away from the "mixed phases" which can contain other parasitic phases such as $\mathrm{La}_{2} \mathrm{Zr}_{2} \mathrm{O}_{7}$ and $\mathrm{La}_{2} \mathrm{Ti}_{2} \mathrm{O}_{7}{ }^{39} \mathrm{It}$ was pointed out in Ref. 39 that the presence of these mixed phases can have a detrimental effect on optical transparency due to the heterogeneous nature of the phase mixture.

In some or all cases, the EO response of the films in the as-grown state was rather weak, ${ }^{17,18}$ probably due to the presence of FE domains with different orientations. A poling step considerably improved the EO response; as previously mentioned, this poling process aligns some or all of the FE domains in the film in a well-defined polar direction. ${ }^{18}$

One stand-out report showed very high EO coefficients of PLZT on (111)-oriented Nb:STO of close to $500 \mathrm{pm} / \mathrm{V}$ for the $(8 / 35 / 65)$ composition. ${ }^{55}$ It should be noted that, however, this work did not address optical guiding, and therefore (given the typical requirement to remove $\mathrm{Zr}$ to increase optical transparency as described above), the optical loss was probably rather high. This highlights a common design problem-optimizing conflicting figures of merit requires trade-offs.

Kang et al. ${ }^{14}$ used a modified ellipsometry technique to measure the EO response of PZT films grown by sputtering on $\mathrm{Nb}: \mathrm{STO}$. An advantage of using this technique was the possibility to separately determine the $r_{13}$ and $r_{33}$ coefficients, rather than an effective coefficient. At $633 \mathrm{~nm}$, the EO coefficients were $r_{33}=-157.1$ and $r_{13}=22 \mathrm{pm} / \mathrm{V}$, while the effective linear and quadratic coefficients were $-134.6 \mathrm{pm} / \mathrm{V}$ and $8.5 \times 10^{-18} \mathrm{~m}^{2} / \mathrm{V}^{2}$, respectively.

To summarize this part on PLZT, typical EO coefficients are of the order of $50-100 \mathrm{pm} / \mathrm{V}$ (Refs. 12, 14, and 54). The most promising substrate appears to have been sapphire; in addition, it enables good mode confinement given the contrast in refractive indices (for further information on waveguiding, the reader is referred to Sec. V of this 
(a)

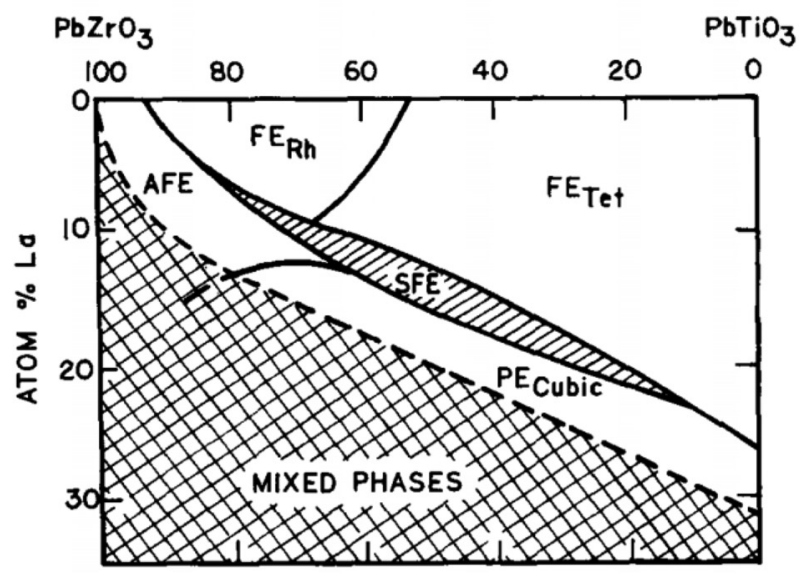

(b)

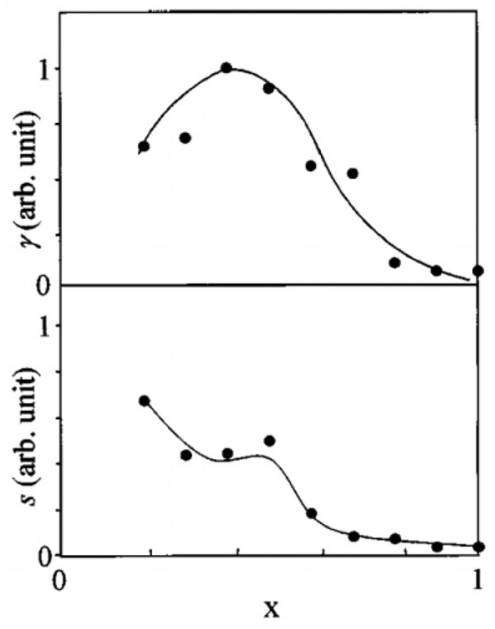

FIG. 3. (a) FE phase diagram of PLZT. Reproduced with permission from G. H. Haertling, Ferroelectrics 75, 25 (1987). Copyright 1987 Taylor \& Francis. (b) Linear (top) and quadratic (bottom) EO response as a function of composition $x$ for $\mathrm{Ba}_{1-x} \mathrm{Sr}_{x} \mathrm{TiO}_{3}$ films. Reproduced with permission from Appl. Phys. Lett. 76, 769 (2000). Copyright 2000 AIP Publishing LLC.

review) and is relatively well lattice-matched to the (111) orientation of PLZT. The interest in PLZT for optical thin film applications appeared to decline in the early 1990s. As fabrication methods improved, thin film epitaxy of more attractive EO materials such as LNO and BTO became possible.

\section{Lithium niobate}

Epitaxial growth of lithium niobate films was considered by numerous groups as early as the $1970 \mathrm{~s} .{ }^{58-60}$ Various growth methods have been used for LNO thin films: MBE (Ref. 58), liquid phase epitaxy, ${ }^{59}$ MOCVD (Refs. 61 and 62), PLD (Refs. 47 and 63-65), sol-gel, ${ }^{66}$ and rf sputtering. ${ }^{67}$ Homoepitaxy (i.e., LNO thin film on the LNO substrate) was an attractive method since lattice mismatch between the film and the substrate is zero, and by modifying the growth conditions, the film's refractive index can be controlled to thus create a guiding layer. ${ }^{58} \mathrm{LiTaO}_{3}$ (LTO) is also an appealing substrate choice ${ }^{61,62}$ since the crystal symmetry is identical and the lattice mismatch is almost zero. A key challenge for LNO thin films has always been obtaining high quality epitaxial growth (particularly regarding stoichiometry) and controlling the crystallographic orientation of the film layer. ${ }^{65,68}$ Furthermore, the existence of complex crystallographic twins causes complications in (i) waveguiding, where optical losses can be high, and (ii) the analysis of the EO effect in such films. For films grown by PLD, stoichiometry can be controlled by laser fluence ${ }^{69}$ and other growth conditions such as oxygen pressure. ${ }^{64,65}$ While some authors required to account for the volatility of lithium by using Li-enriched targets ${ }^{70}$ others have shown that it is possible to use stoichiometric targets and a very high laser fluence (and hence growth rate) to obtain stoichiometric films. ${ }^{64}$ To control the crystallographic orientation, the oxygen growth pressure has been shown to play a role, possibly due to the oxygen stacking arrangements for different orientations. ${ }^{47}$

Despite these growth challenges, there are numerous reports of the EO characterization of epitaxial LNO films grown on various substrates and by various methods. As early as 1973, the EO response of an LNO thin film (fabricated by "epitaxial growth by melting"-EGM) was reported. ${ }^{71}$ The $r_{13}$ and $r_{33}$ coefficients were obtained and found to be lower (2.3 and $12 \mathrm{pm} / \mathrm{V}$, respectively) than the values typically found in LNO bulk (cf. Table I); this was suggested to be due to the lower crystalline quality of the film. The optical properties of LNO epitaxial films were explored more extensively in the mid-late 1990s. ${ }^{72}$ A review of work pre-1995 can be found in Ref. 72; here, we focus on important results and some newer work. Propagation losses of waveguide structures were found to be as low as $0.9 \mathrm{~dB} / \mathrm{cm}$ for LNO on sapphire substrates. ${ }^{72}$

Lee et al. showed that the EO response of LNO films grown on sapphire substrates depends strongly on the growth behavior and substrate orientation. ${ }^{47}$ Notably, for LNO on $\mathrm{Al}_{2} \mathrm{O}_{3}$ (0001), epitaxial LNO (0001) films were obtained. On the other hand, when using $\mathrm{Al}_{2} \mathrm{O}_{3}(11 \overline{2} 0)$ substrates, either LNO (0001) or LNO (11 $\overline{2} 0)$ was stabilized, depending on the oxygen growth pressure. As mentioned above, this dependence on pressure is likely related to the different oxygen stacking arrangements. The EO effect in these two different orientations of $\mathrm{LNO}$ on $\mathrm{Al}_{2} \mathrm{O}_{3}(11 \overline{2} 0)$ was measured by the Senarmont method. For the LNO (0001) film, no change in the birefringence with applied electric field was measured [Fig. 1(c)], indicating a very weak EO effect, attributed to the crystallographic symmetry of the LNO layer. On the other hand, the $(11 \overline{2} 0)$ LNO film showed a large quadratic EO coefficient of $2.38 \times 10^{-15}(\mathrm{~m} / \mathrm{V})^{2}$, as shown in Fig. 1(c). This very large effect was not fully understood since the transition temperature of LNO is very high when compared to other FE materials with large EO responses. Nonetheless, the large quadratic EO effect was suggested to be attractive for devices, ${ }^{47}$ since it removes the need for single-domain films and thus relaxes fabrication requirements.

It was realized by the end of the 1990s that, due to the growth difficulties and complex twinning structures in LNO films, a "simpler" material was desired in order to make further progress in integrated thin film optical devices. ${ }^{73}$ At that 
time, the perspective for $\mathrm{BaTiO}_{3}$ (BTO) thin films was more favorable, due to its larger EO coefficients, simpler perovskite structure, and relative ease of fabrication. Section IIE introduces BTO as the active layer for EO thin film devices.

\section{E. Barium titanate and related compounds}

Barium titanate $\left(\mathrm{BaTiO}_{3}-\mathrm{BTO}\right)$ is the prototypical tetragonal ferroelectric and has been used for a long time as a dielectric material in capacitors. In bulk, it has a polarization (at room temperature) of $25 \mu \mathrm{C} / \mathrm{cm}^{2}$ and very large EO coefficients (e.g., $r_{42}=1640 \mathrm{pm} / \mathrm{V}$; Table I). The huge EO coefficients have understandably made it the material of choice for thin-film EO devices since, in principle, the operation voltage can be lower, and the devices can be smaller. The first issue to be overcome was the successful growth of BTO epitaxial thin films. The main issue is the surface roughness, presence of defects (such as hydrogen complexes ${ }^{74}$ ), and domain boundaries that cause significant scattering in waveguide structures. The growth methods mostly employed for film growth of BTO have traditionally been MOCVD and oxide MBE. While high quality $c$-axis (parallel to the polarization) oriented BTO films can be grown by PLD on STO substrates, ${ }^{75}$ low dielectric substrates, such as $\mathrm{MgO}$, are more attractive for EO applications. BTO films have been grown on MgO by MOCVD (Ref. 43), PLD (Refs. 76-78), in some cases up to almost $1 \mu \mathrm{m}$ thick with surface roughness remarkably only $1.1 \mathrm{~nm} .^{76}$ Growth conditions such as the laser repetition rate for PLD have significant influence on the structural quality of the films, ${ }^{78}$ and therefore growth conditions require careful optimization. Integration of BTO films on $\mathrm{Si}$ has allowed this material to take a front row seat in the quest for high performance EO modulators. We discuss such prototype devices in Sec. V.

Petraru et al. showed that the growth conditions can control the orientation of the BTO thin film grown on $\mathrm{MgO}$ substrates. ${ }^{79}$ Using PLD growth, they showed that high laser energy forms $c$-oriented BTO films, while for lower laser energy of $300 \mathrm{~mJ} /$ pulse $a$-oriented (perpendicular to the polarization), BTO films were obtained. The exact mechanism of this growth rate dependence on the orientation is unclear. Using the $a$-oriented film, the authors then demonstrated an effective linear EO coefficient of $r_{\text {eff }}=734 \mathrm{pm} / \mathrm{V}$ in a modulator configuration. The half-wave voltage $V_{\pi}$ was found to be as low as $6.3 \mathrm{~V}$ at $633 \mathrm{~nm}$ and $9.5 \mathrm{~V}$ at $1550 \mathrm{~nm}$.

BTO doped with Fe shows interesting behavior relevant to implementation in optical isolators. For example, Faraday rotation and a weak magnetic moment was reported for $\mathrm{Fe}$ doped BTO by Rajamani et al. ${ }^{80}$ Such a development could be promising as commonly used materials, such as garnets, are not readily integrable with $\mathrm{Si}$.

Another attractive material for electro-optical activity is the solid solution of $\mathrm{BaTiO}_{3}$ and $\mathrm{SrTiO}_{3}-\mathrm{Ba}_{1-\mathrm{x}} \mathrm{Sr}_{\mathrm{x}} \mathrm{TiO}_{3}$ (BST). Initially attractive for microwave applications by virtue of its large dielectric tunability in the microwave frequency range, BST was first considered for its EO response in 2000 by Li et al. (Ref. 81). The initial work required the determination of the optimal concentration for the EO response, and it was found through a combinatorial pulsed laser deposition growth method that the optimal composition for the linear EO effect was for $x=0.3$ [Fig. 3(b)]. Such a composition corresponds to the transition between cubic and tetragonal phases at room temperature.

A detailed study of BST ( $x=0.3)$ films was reported by Wang et al. (Ref. 2). Growth by PLD yielded epitaxial films of reasonable crystalline and surface quality, with a bandgap of $3.5 \mathrm{eV}$. Using the prism-coupling method, the losses [defined as $10 \log _{10}$ (output power/input power)] of $620 \mathrm{~nm}$ thick BST//MgO were $\sim 3 \mathrm{~dB} / \mathrm{cm}$ at $633 \mathrm{~nm}$ and $\sim 1.3 \mathrm{~dB} / \mathrm{cm}$ at $1.55 \mu \mathrm{m}$. Such values, particularly at $1.55 \mu \mathrm{m}$, are well within limits required for integrated optics applications. The quadratic EO coefficient was $6.64 \times 10^{-18}(\mathrm{~m} / \mathrm{V})^{2}$, similar to PZT films, ${ }^{82}$ but smaller than the value $R_{c}=1.0 \times 10^{-14} \mathrm{~m}^{2} /$ $\mathrm{V}^{2}$ for BST $(x=0.4)$ on $\mathrm{MgO}$ obtained by Kim et al. ${ }^{83} \mathrm{a}$ few years earlier.

The structure, optical properties, and EO response of BSTO $(x=0.3)$ films grown on $\mathrm{LaAlO}_{3}$ (LAO), (La,Sr) $(\mathrm{Al}, \mathrm{Ta}) \mathrm{O}_{3}$ (LSAT), and STO substrates were investigated by Wang et al. to try to ascertain the influence of strain on the properties. ${ }^{11}$ (Note that Sec. III discusses strain effects in more detail.) Although the nominal misfit compressive strain imposed on BSTO by LAO, LSAT, and STO are $-4.8 \%$, $-2.6 \%$, and $-1.7 \%$, respectively, only the film on STO exhibited a structure consistent with an in-plane compressive strain (of about $-0.8 \%$ ), while the films on LAO and LSAT surprisingly, and counterintuitively, showed an elongated in plane lattice constant (the out of plane lattice constant appeared to be comparable to bulk BST). The latter point was attributed by the authors to a likely oxygen stoichiometry difference in these films. Interestingly, the strain state induced (or not) by the substrate influenced the dielectric response, and thus the electro-optic effect. The film grown on STO was not ferroelectric and therefore did not exhibit EO modulation, while the films on LAO and LSAT showed a linear EO behavior, consistent with their ferroelectric character, as evidenced by dielectric measurements. The effective linear EO coefficient for BST//LSAT was the largest of the three samples at about $125 \mathrm{pm} / \mathrm{V}$.

\section{F. Bismuth ferrite}

Bismuth ferrite $\left(\mathrm{BiFeO}_{3}-\mathrm{BFO}\right)$ has the same crystallographic symmetry as LNO and is probably the most recent ferroelectric to be considered for thin film EO applications. $\mathrm{BFO}$ is multiferroic-meaning that it has coexisting ferroic orders: in this case, strong ferroelectricity ${ }^{84}$ is combined with antiferromagnetic order. ${ }^{85}$ The EO response of BFO was first considered in single-domain (111)- and (110)-oriented films, upon which reflection and transmission geometry measurements were performed, respectively. ${ }^{20}$ The EO coefficients were found to be modest at $r_{\text {eff }}=12 \mathrm{pm} / \mathrm{V}$ (somewhat surprising given the very large polarization of BFO); however, taking into account the rather low dielectric constant ${ }^{36}$ and high refractive index ${ }^{86}$ of BFO, the figure of merit $n^{3} r / \varepsilon=8.0$ (Table I) makes it as performant as some other ferroelectrics such as LTO. A waveguide modulator prototype has been demonstrated using BFO films on STO; this is described in Sec. V. 
In the late $2000 \mathrm{~s}$, it was found that the optical bandgap of BFO_which had been successfully fabricated in epitaxial thin films a few years earlier ${ }^{87}$ - was $2.7 \mathrm{eV}$, placing it in the blue region of the visible spectral range. This triggered widespread interest in this material as a material for energy harvesting (most other ferroelectrics have bandgaps that are much higher at $>3.2 \mathrm{eV}$ ). In 2009, it was found that BFO exhibited the so-called bulk photovoltaic effect (BPV) and subsequently that large photovoltages could be generated from BFO thin films. ${ }^{88,89}$ This is further discussed in Sec. IV of this review.

\section{G. Other ferroelectric films for EO modulation}

Other ferroelectric materials, such as $\mathrm{LTO}, \mathrm{KNbO}_{3}$ (KNO), $\mathrm{KTa}_{x} \mathrm{Nb}_{1-x} \mathrm{O}_{3}$ (KTN), $(\mathrm{Sr}, \mathrm{Ba}) \mathrm{Nb}_{2} \mathrm{O}_{6}(\mathrm{SBN}),{ }^{82}$ and $\mathrm{Pb}\left(\mathrm{Mg}_{1 / 3} \mathrm{Nb}_{2 / 3}\right) \mathrm{O}_{3}-\mathrm{PbTiO}_{3}$ (PMN-PT) (Ref. 90), have been explored for their EO effects in epitaxial thin films. KTN films epitaxially grown on $\mathrm{MgAl}_{2} \mathrm{O}_{4}$ (magnesium aluminate spinel-MAS) substrates were measured by Hoerman et $a l .{ }^{91}$ and shown to possess composition-sensitive EO coefficients. The best coefficients of about $40 \mathrm{pm} / \mathrm{V}$ were obtained at $x=0.2$ and 0.65 , the latter being due to its vicinity to the ferroelectric-paraelectric phase boundary at $x=0.7$. KNO grown on $\mathrm{MgO}$ was investigated by Beckers et al. where it was required to use a buffer of BTO to promote epitaxial growth. ${ }^{76}$ PMN-PT on $\mathrm{MgO}$ and LAO was explored by Lu et al., ${ }^{90}$ where it was found that the quadratic EO effect was $0.75 \times 10^{-16}(\mathrm{~m} / \mathrm{V})^{2}$, and propagation loss was $4 \mathrm{~dB} / \mathrm{cm}$. Finally, very large EO coefficients of up to $844 \mathrm{pm} / \mathrm{V}$ were observed in SBN films grown on $\mathrm{MgO} .{ }^{92}$ Although rather large, such coefficients are still smaller than the corresponding bulk values for SBN.

Interestingly, $\mathrm{Lu}$ and $\mathrm{Knize}^{93}$ explored the dielectric and EO response of PMN-PT superlattices and found that the dielectric constant was improved, and EO effect increased by an order of magnitude, by virtue of the interlayer coupling in the superlattices. Such work highlights that strain and interfacial effects can both be used to tailor the EO response for optical purposes.

Regardless of the material system, an important aspect is the fact that epitaxial strain has a significant bearing on the functional response. Therefore, Sec. III is devoted to the understanding of strain effects on optical properties.

\section{STRAIN MODULATION OF OPTICAL PARAMETERS}

\section{A. Strain engineering}

When a film is epitaxially grown on a single-crystal substrate of a different material (heteroepitaxy), a misfit strain exists between the film and substrate, related to their respective bulk structures. The commercial availability of a wide range of substrate crystals suitable for oxide heteroepitaxy has allowed considerable levels of strain to be imparted on the grown films. 94 This technique, known as "strain engineering" [Fig. 4]-i.e., using epitaxial strain from the underlying substrate to transform the structure and functional response of the film-offers exciting possibilities. Examples include creating ferroelectric phases of nominally (a)

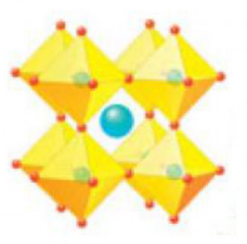

(b)

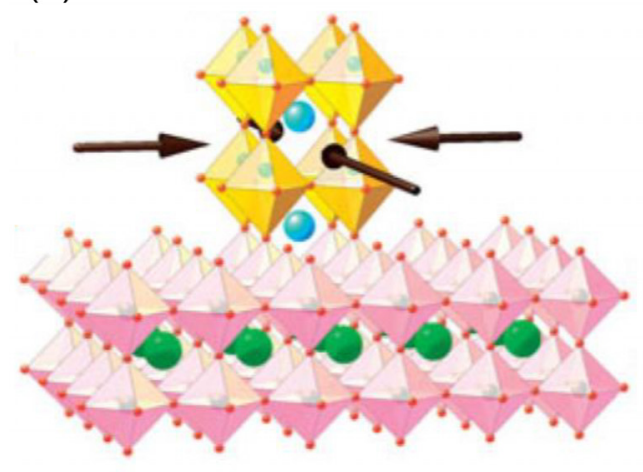

(c)

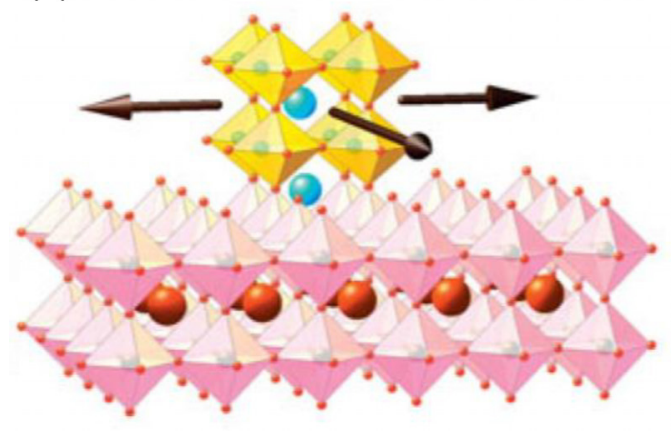

FIG. 4. Concept of epitaxy of an $\mathrm{ABO}_{3}$ perovskite unit cell (a). Large strains can be applied to epitaxial thin films by strain engineering, by applying either compressive (b) or tensile (c) strain from the underlying single-crystal substrate. Reproduced with permission from D. G. Schlom et al., MRS Bull. 39, 118 (2014). Copyright 2014 Cambridge University Press.

paraelectric materials, such as $\mathrm{STO}$ or $\mathrm{KTaO}_{3}$ (KTO) (Refs. 95-97), inducing large variations of ferroelectric and magnetic transition temperatures, ${ }^{98,99}$ changes in spin order, ${ }^{85}$ or generating new phases of existing compounds (such as the so-called T-like phase of BFO; Refs. 100 and 101).

The number line shown in Fig. 5(a) indicates the pseudo-cubic lattice parameter (where applicable) for substrates and the thin films that we discuss here. While the influence of the possible strains using such substrates on magnetism and ferroelectricity has been well covered in recent years, the effect on optical properties is comparatively unexplored. The strain modulation of the optical response is of considerable interest because one can envisage giant EO responses which would be beneficial to the downscaling of optical devices. Furthermore, changes in the bandgap and optical constants (i.e., the complex refractive index) due to the imposed strain can yield new properties that may not be available in the corresponding bulk phase.

Important characteristics (bandgap, refractive index, and dielectric constant) of materials used for substrates and FE 
(a)

Lattice parameter

(A)

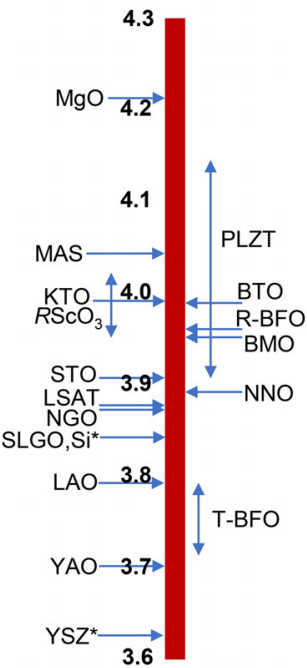

(b)

Optical

band gap

(eV) (c)

$n$ at

$633 \mathrm{~nm}$ (d)

Dielectric

constant
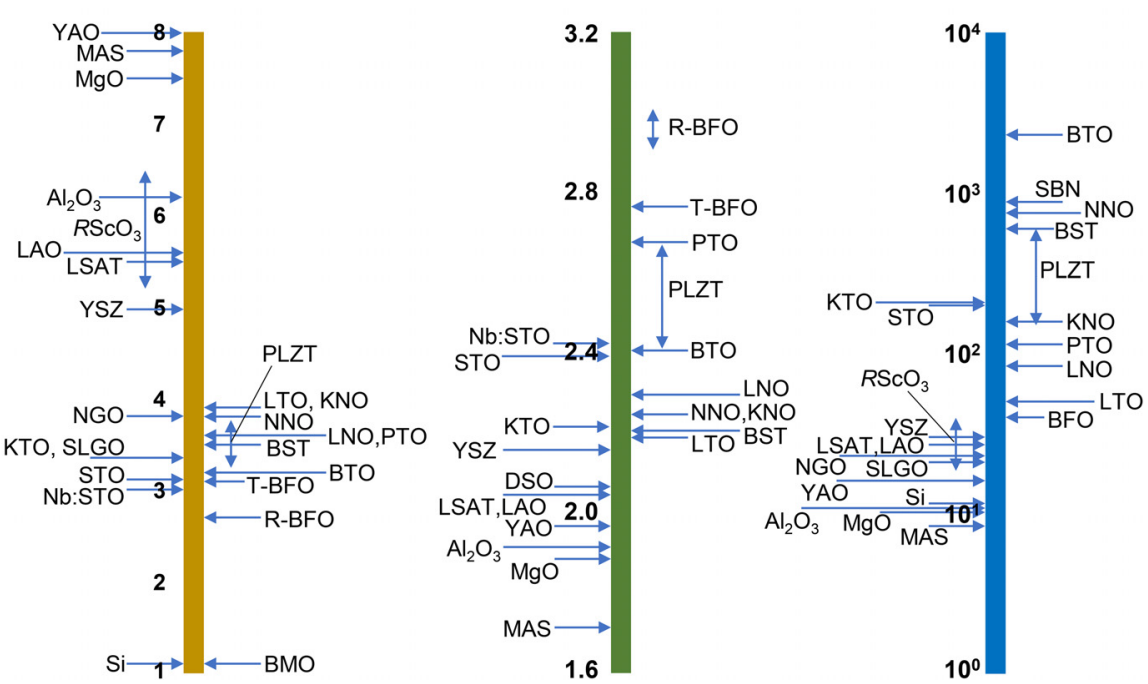

FIG. 5. Characteristics of substrates and films appropriate for optical applications. On the left of each number line are (typical) substrates; while on the right are (typical) films (inspired by and adapted from Ref. 94). These number lines give information relevant to (a) crystal lattice mismatch, (b) bandgap determination of a film (by transmittance), (c) optical waveguiding, and (d) microwave phase matching. (* denotes that the lattice constant is shown here for $45^{\circ}$ epitaxy, i.e., the substrate lattice constant is a factor of $\sqrt{2}$ larger than the value shown here.)

thin films are plotted in Figs. 5(b)-5(d). The relevance of these parameters in the context of optical applications will become apparent later. Section III B is devoted to the understanding of strain effects in thin film ferroelectrics and focuses on how novel responses can be predicted in such systems, using $\mathrm{PbTiO}_{3}$ films as a representative example.

\section{B. Theoretical approaches for strain modulated optical parameters}

We first consider the theoretical concepts governing the strain-modulated optical response of FE oxides. Since optical properties are intimately related to the electronic band structure of materials, it is important to begin with a good understanding of the band structure.

A commonly adopted approach for determining the optical properties, namely, the complex dielectric function $\varepsilon^{\prime}$ $+i \varepsilon^{\prime \prime}$ and complex refractive index $n+i k$, is to use density functional theory (DFT) to determine the band structure. Often DFT is used with approximations to the exchangecorrelation energy functional, ${ }^{102}$ such as local density approximations (LDA), generalized gradient approximations (GGA), and hybrid functionals. Hybrid functionals, such as the Heyd-Scuseria-Ernzerhof (HSE) exchange-correlation functional, ${ }^{103}$ usually give more accurate results, albeit at a much higher computational cost. After the electronic band structure calculations, the imaginary part of the dielectric tensor can be obtained by

$$
\begin{aligned}
\varepsilon_{\alpha \beta}^{\prime \prime}(\omega)= & \frac{4 \pi^{2} e^{2}}{\Omega} \lim _{q \rightarrow 0} \frac{1}{q^{2}} \sum_{c, v, \boldsymbol{k}} 2 \omega_{\boldsymbol{k}} \delta\left(\epsilon_{c \boldsymbol{k}}-\epsilon_{v \boldsymbol{k}}-\omega\right) \\
& \times\left\langle u_{c \boldsymbol{k}+\boldsymbol{e}_{\alpha} q} \mid u_{v \boldsymbol{k}}\right\rangle\left\langle u_{c \boldsymbol{k}+e_{\beta} q} \mid u_{v \boldsymbol{k}}\right\rangle^{*}
\end{aligned}
$$

where the indices $c$ and $v$ refer to conduction and valence band states, respectively, $u_{c k}$ is the cell periodic part of the orbitals at the $k$-point $\boldsymbol{k}$, and $\boldsymbol{e}_{\alpha}$ is a unit vector along the $\alpha$ Cartesian direction. ${ }^{104}$ Finally, the real part of the dielectric tensor $\varepsilon_{\alpha \beta}^{\prime}$ can be obtained through the Kramers-Kronig transformation

$$
\varepsilon_{\alpha \beta}^{\prime}(\omega)=1+\frac{2}{\pi} P \int_{0}^{\infty} \frac{\varepsilon_{\alpha \beta}^{\prime \prime}\left(\omega^{\prime}\right) \omega^{\prime}}{\omega^{\prime 2}-\omega^{2}} d \omega^{\prime},
$$

where $P$ denotes the principal value. We then obtain the complex refractive index using

$$
\tilde{\varepsilon}=\varepsilon^{\prime}+\mathrm{i} \varepsilon^{\prime \prime}=(n+i k)^{2},
$$

where $k$ is the extinction coefficient and $n$ is the refractive index. (Note that the general case is $\tilde{\varepsilon} \cdot \tilde{\mu}=\varepsilon^{\prime}+\mathrm{i} \varepsilon^{\prime \prime}=(n+i k)^{2}$, where $\mu$ is the magnetic permeability. Here, since we deal with non-magnetic materials we consider $\mu=1$.)

Electronic band structure calculations show that the band structure can be modified using, for instance, strain. As shown in Fig. 6(a), both tensile strain and compressive strain increase the electronic bandgap in the $R$-like phase of $\mathrm{BiFeO}_{3}$ [more clearly demonstrated in the inset of Fig. 6(a)]. At zero strain, the $\mathrm{Fe} d_{x y}, d_{y z}$, and $d_{x z}$ orbitals are degenerate near the Fermi level. ${ }^{86}$ The tensile and compressive straininduced change in oxygen octahedra rotation and polar mode slightly lift the degeneracy of the Fe $3 \mathrm{~d}$ orbitals, increasing the bandgap. The $T$-like phase (stabilized, when compared to the $R$-like phase, by large compressive strain) shows a decrease in the electronic bandgap when compared to the $R$-like phase, as shown in Fig. 6(b). This effect arises from the pyramidal coordination of $\mathrm{FeO}_{5}$ in this highly elongated $T$-like phase which induces a large splitting in the $3 \mathrm{~d}$ 
(a)

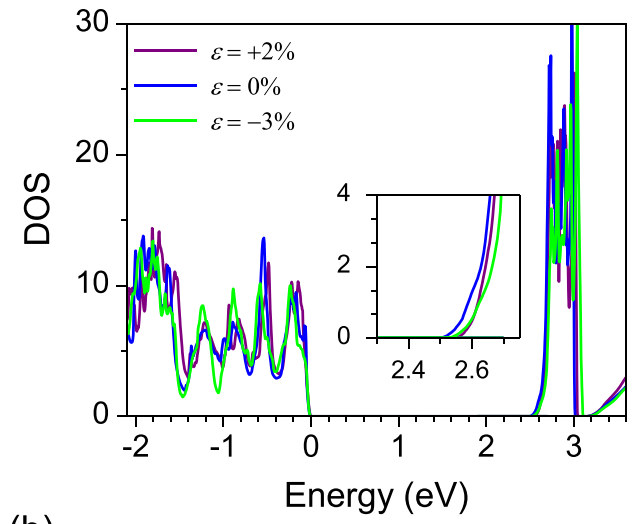

(b)

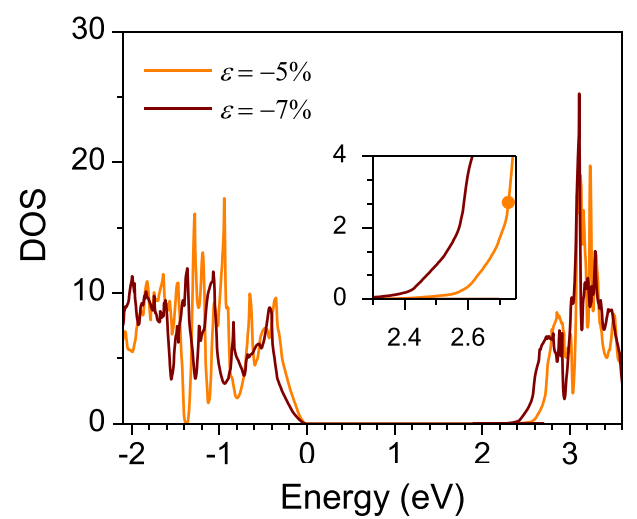

(c)

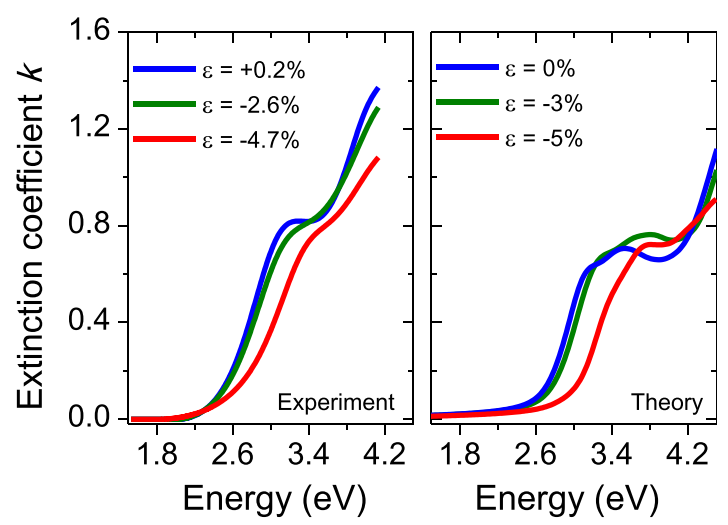

(d)
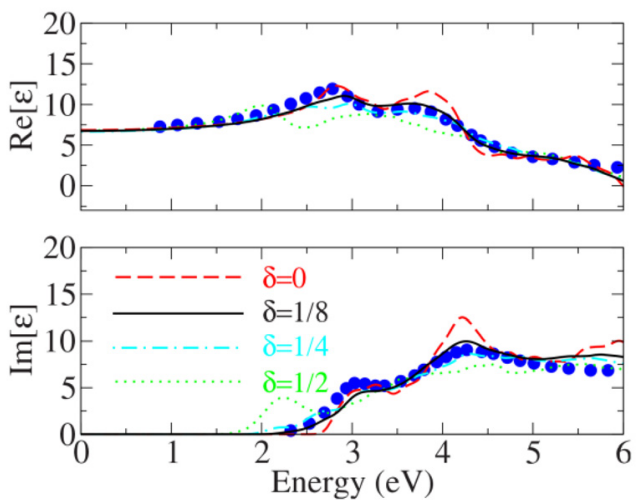

FIG. 6. First-principles calculations of optical properties. Total density of states (DOS) for the $R$-like (a) and $T$-like (b) phases of BFO. The insets show the conduction band edges. Note that strain modifies the band structure and DOS, which induces a change in the bandgap (from Ref. 86). (c) Experimental (left) and calculated (right) extinction coefficient $k$ for BFO films under various levels of epitaxial strain (from Ref. 86). (d) Real (top) and imaginary (bottom) parts of the dielectric function for $\mathrm{BFO}$ for different levels of oxygen vacancies. Note that the form of the curves is strongly dependent on the concentration of oxygen vacancies, and the best match with experiment (blue circles) is found for $\delta=1 / 8$. Reproduced with permission from Appl. Phys. Lett. 95, 231906 (2009). Copyright 2009 AIP Publishing LLC.

orbitals, with the $d_{x y}$ orbital lowering in energy. ${ }^{86}$ It should be pointed out, however, that although the electronic bandgap is lower in the $T$-like phase, the optical bandgap (which is measured by ellipsometry and other techniques ${ }^{105,106}$ ) actually increases, due to the transitions between the valence band maximum (predominantly $\mathrm{O} 2 \mathrm{p}$ in character), and this $\mathrm{Fe} d_{x y}$ orbital being rather weak. ${ }^{86}$ The behavior of the optical response as a function of strain is summarized in Fig. 6(c) where the experimental and theoretical extinction coefficient is plotted. The agreement here regarding the band edge is rather good; and the higher optical bandgap of the $T$-like phase is evident.

First principles techniques can also be used to estimate the influence of defects on the optical response. For example, in Fig. 6(d), the complex dielectric function is shown for BFO films as a function of the oxygen vacancy concentration. ${ }^{107}$ Interestingly, the calculations show the best match with experimental data when the vacancy concentration is about $1 / 8$ (i.e., $12.5 \%$ ); suggesting that the films used in the experimental study ${ }^{108}$ may have had intrinsically a relatively high oxygen vacancy density.

\section{Large elasto-optic effects in ferroelectric thin films}

The elasto-optic effect describes the change in permittivity, $\varepsilon$, due to mechanical strain, $\eta$. It is useful for creating or designing acousto-optic modulators, acousto-optic tunable filters, and electro-optic deflectors. ${ }^{109}$ This effect is quantified by the elasto-optic tensor, $p_{i j}$, defined as

$$
\Delta\left(\frac{1}{\tilde{\varepsilon}}\right)_{i}=\sum_{j} p_{i j} \eta_{j}
$$

where $i$ are $j$ are both integers that can vary from 1 to 6 (using Voigt notation). Using the extinction coefficient $k$ and refractive index $n$ tensors through Eq. (6) and considering the low-frequency field (the Pockels effect), $\varepsilon^{\prime \prime}$ is about zero, and Eq. (7) can be rewritten as

$$
\Delta\left(\frac{1}{n^{2}}\right)_{i}=\sum_{j} p_{i j} \eta_{j} .
$$

The above equation (8) is primarily used to compute the elasto-optic coefficient. Note that, as indicated above, both refractive index $n$ and strain $\eta$ have 6 components (in Voigt notation), resulting in 36 elasto-optic coefficients $p_{i j}$. However, common elasto-optic materials (such as $\mathrm{SiO}_{2}$ and $\mathrm{LiNbO}_{3}$ ) have relatively high symmetry, and as a result, only a few of the elasto-optic tensor components are non-zero. For a full discussion of the computation methods and details used for the prediction of the elasto-optic properties, the reader is referred to Ref. 110. 
Briefly here, the strain tensor components of " 1 " and "2" (with the " 1 ," "2," and " 3 " denoting the pseudo-cubic [100], [010], and [001] directions, respectively) were considered, and the condition $\eta_{1}=\eta_{2}$ was imposed to mimic epitaxial conditions. The resulting effective elasto-optic coefficients are thus obtained from

$$
\begin{gathered}
{\left[1 / n_{1}^{2}\left(\eta_{1}\right)\right]-\left[1 / n_{1}^{2}(0)\right]=\left(p_{11}+p_{12}\right) \eta_{1},} \\
{\left[1 / n_{2}^{2}\left(\eta_{1}\right)\right]-\left[1 / n_{2}^{2}(0)\right]=\left(p_{21}+p_{22}\right) \eta_{1}, \quad \text { and }} \\
{\left[1 / n_{3}^{2}\left(\eta_{1}\right)\right]-\left[1 / n_{3}^{2}(0)\right]=\left(p_{31}+p_{32}\right) \eta_{1} .}
\end{gathered}
$$

In tetragonal $(\mathrm{T})$, orthorhombic $(\mathrm{O})$, rhombohedral $(\mathrm{R})$, and monoclinic $C m$ phases of epitaxial $\mathrm{PbTiO}_{3}$ films (see the following text), $n_{1}=n_{2}=n_{o}$, the ordinary refractive index, and $n_{3}=n_{e}$, which is the extraordinary refractive index. In the monoclinic $P m$ phase, $n_{1} \neq n_{2}$. This first-principles method of computing the elasto-optic coefficients has the desired accuracy; as an example for bulk $\mathrm{LiNbO}_{3}$ (using the hexagonal setting), the computed elasto-optic coefficient is $p_{31}=0.17$, which compares well with the corresponding experimental value of 0.18 (Ref. 111).

\section{Elasto-optic effects in ferroelectric oxide thin films- $-\mathrm{PbTiO}_{3}$ as a test case}

Chen et al. ${ }^{112}$ theoretically investigated the influence of strain on the optical index $n$ of epitaxial PTO films and thus derived the elasto-optic effect in this material. Having ensured that their computations accurately predicted the ground state of PTO without strain, they then showed the stability of various phases as a function of in-plane tensile strain. For strains ranging from $0.75 \%$ to $1.75 \%$, a monoclinic $\mathrm{Cm}$ phase (with energy close to that of the $T$ phase) becomes the ground state [Fig. 7(a)]. Interestingly, it was found that another monoclinic $P m$ phase exhibits a very similar energy to the $C m$ phase, indicating that both these phases can likely be stabilized in PTO films by, e.g., strain engineering and/or electric field. Practically, both $\mathrm{Pm}$ and $\mathrm{Cm}$ phases have been observed for ultrathin PTO films grown on $\mathrm{DyScO}_{3}$, a substrate that imparts a $1.4 \%$ biaxial strain. ${ }^{113}$

As shown in Fig. 7(b), the predictions found that the $\mathrm{Cm}$ phase decreases its axial ratio from 1.022 to 0.988 when the epitaxial strain ranges from $0.75 \%$ to $1.75 \%$. In this tensile strain range, the $\mathrm{Cm}$ phase has both out-of-plane and inplane components of polarization, implying that a polarization rotation phenomenon takes place. In contrast, note that at zero strain the ground state of PTO has polarization only along the $c$-axis. When this in-plane strain increases from $0.75 \%$ to $1.75 \%$, the in-plane polarization $P_{x}$ of $C m$ increases from about zero to $0.66 \mathrm{C} / \mathrm{cm}^{2}$, while the out-of-plane polarization $P_{z}$ commensurately decreases from $0.75 \mathrm{C} / \mathrm{cm}^{2}$ to almost zero. As shown in Fig. 7(c), the polarization of the $\mathrm{Cm}$ phase continuously rotates from the out-of-plane [001] direction toward the in-plane [110] direction when increasing strain in this intermediate range.

For the monoclinic Pm phase, the tetragonality (i.e., $c / a$ ratio) decreases to less than 1 as the imposed strain (a)

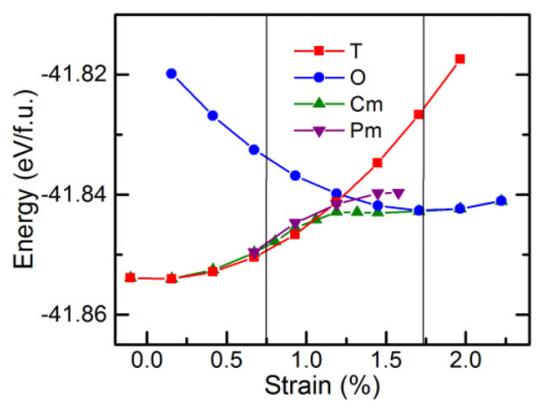

(b)

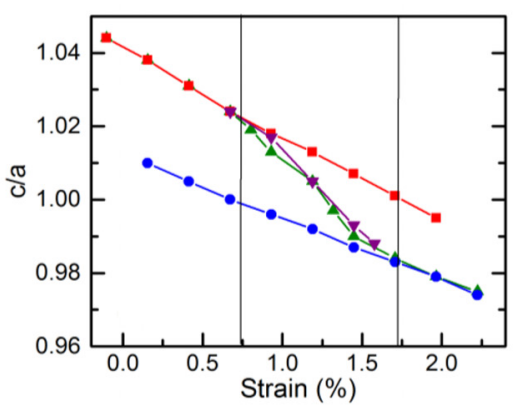

(c)

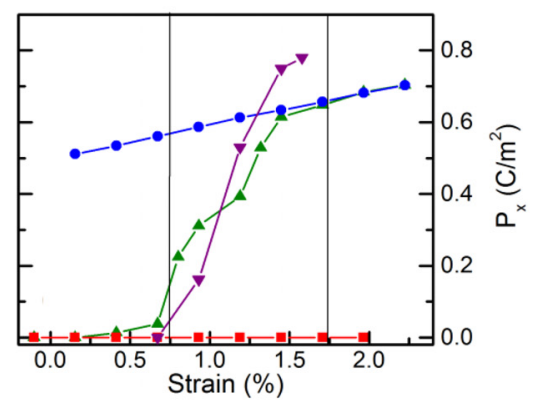

(d)

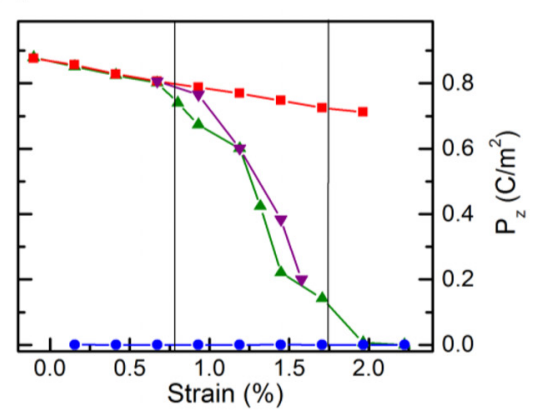

(e)

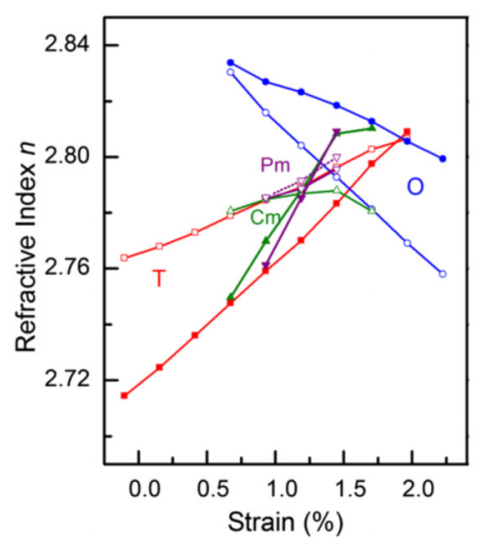

(f)

(g)

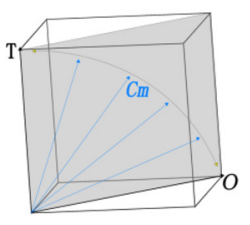

FIG. 7. First principles calculations for various phases of $\mathrm{PbTiO}_{3}$ under tensile strain. Strain-dependent properties for the tetragonal $(T)$, orthorhombic $(O)$, and $\mathrm{Cm}$ and $\mathrm{Pm}$ monoclinic phases: (a) total energy, showing stability regions; (b) axial ratio $c / a$; (c) in plane polarization $P_{x}$; and (d) out of plane polarization $P_{z}$. (e) refractive index $n$ for the four considered phases. Note that for $P m$ and $C m$ phases the change in $n$ with strain is large, yielding large elasto-optic coefficients (see the text). Schematic representations of polarization directions of the $C m, P m, O$, and $T$ phases. Polarization of the $T$ phase is along the [001] direction, of the $O$ phase is along the [110] direction. Polarization of $\mathrm{Cm}$ is along a [uuv] direction (with $u$ different from $v$ ), which rotates from the [100] direction to the [110] direction under strain, and the polarization of $P m$ is along a [u0v] direction (with $u$ different from $v$ ), which rotates from the [001] direction to the [100] direction under strain. Adapted from Refs. 110 and 112. 
progresses from $0.75 \%$ to $1.75 \%$. Thus, the axial ratio of the $\mathrm{Pm}$ phase behaves qualitatively (and even quantitatively) very similarly to that of the $C m$ phase. Figures 7(c) and 7(d) show how the $P_{z}$ of $P m$ decreases and $P_{x}$ increases in this tensile strain range. Note that $P_{y}=0$ for the $P m$ phase, which differs from the case of the $\mathrm{Cm}$ phase, for which $P_{y}=P_{x}$ is non-zero. Figures 7(f) and 7(g) schematically depict how the polarization of the $\mathrm{Pm}$ phase rotates from the pseudo-cubic [001] to [100] directions upon increasing tensile strain. As shown next, these varying polarization rotation paths induce markedly distinct optical properties and elasto-optic effects in the $\mathrm{Cm}$ and $\mathrm{Pm}$ phases.

The optical properties and elasto-optic effect indeed are rather unique in PTO films when strain is applied. The refractive index properties were discussed in detail in Ref. 112. Briefly, it was found that there exists a hierarchy between the ordinary $\left(n_{\mathrm{o}}\right)$ and extraordinary $\left(n_{\mathrm{e}}\right)$ refractive index as a function of the tetragonality. As tetragonality is tuned by the imposed strain, it follows that the optical refractive index is also strain-sensitive.

This strong strain sensitivity implies that the elasto-optic coefficients in such monoclinic PTO phases are rather large. The calculations indeed found that the effective elasto-optic coefficient $\left(p_{31}+p_{32}\right) / 2$ for the rotational low-symmetry phases, namely, monoclinic $\mathrm{Cm}$ and $\mathrm{Pm}$ phases, are very large. These coefficients amount to $200 \%$ those of the $T$ phase and $300 \%$ those of the $O$ phase. This large elasto-optic coefficient can be linked to $n_{\mathrm{e}}$ in $\mathrm{Cm}$ and $n_{3}$ in $\mathrm{Pm}$, which are extremely sensitive to the applied strain. They are the largest elasto-optic coefficients predicted for this family of ferroelectric oxides. For instance, they are about twice as large as the coefficient of lithium niobate, for which $p_{31}=0.18$, and $30 \%-60 \%$ bigger than that of quartz $\left(p_{31}=0.29\right)$ (Ref. 111).

The preceding discussion therefore offers a new route toward the discovery of large elasto-optic effect in ferroelectric oxide thin films materials by strain engineering. It essentially involves identifying low-symmetry phases that undergo a rapid evolution of the axial ratio and polarization with strain. This rapid evolution can occur in epitaxial ferroelectric films ${ }^{114,115}$ and the morphotropic phase boundaries of some solid solutions ${ }^{116-118}$ when varying the composition or hydrostatic pressure. Finally, large elasto-optic coefficients have been demonstrated in PTO thin films, ${ }^{119}$ and in multiferroic $\mathrm{BiFeO}_{3}$ (Refs. 86 and 120).

\section{E. Experimental reports on strain-modulated optical properties}

Having shown that theoretical methods can predict novel optical responses with strain, we now turn to a discussion of experimental findings related to strain-modulated optical properties. The most common technique to measure the optical properties (e.g., complex refractive index and bandgap) of thin films is spectroscopic ellipsometry, ${ }^{121,122}$ a routine characterization method. The experimental results in this section thus rely predominantly on measurements conducted by ellipsometry.

Early reports of the strain-modulated optical properties of thin film oxides focus mostly on materials such as STO.
Applying strain to STO had earlier yielded novel phases such as induced ferroelectricity ${ }^{97}$ and so it was expected that optical properties should also be modified. Ultrathin $(\sim 15 \mathrm{~nm})$ films of STO were grown under various levels of strain on $\mathrm{LAO}_{,} \mathrm{DyScO}_{3}$ (DSO), KTO, and $\mathrm{MgO}$ substrates, and their optical response measured by ellipsometry. ${ }^{123}$ Even though strain appeared to modify the optical response by modifying interband transitions through a "strain-induced polarization," there appeared not to be a clear systematic effect. More detailed work on STO//KTO, under $\sim 2 \%$ tensile strain, showed that the absorption edge was shifted toward higher energies, ${ }^{124}$ which interestingly is in agreement (at least in sign) with the shift in bandgap predicted by DFT theory of strained STO (this is described in Sec. IIIF). ${ }^{125}$ Nevertheless, it should be pointed out that oxygen vacancies can also induce a perceived decrease in bandgap ${ }^{126}$ by smearing the fundamental edge - such an observation implies that sufficient caution be exercised when interpreting strain-induced modifications of the optical response.

Moving now to ferroelectric films, Tyunina et al. ${ }^{127}$ grew $\mathrm{KTO}, \mathrm{KNO}$, and $\mathrm{NaNbO}_{3}$ (NNO) films on STO substrates and found that the crystal symmetry of the films plays an important role regarding how the bandgap changes. For KTO//STO (strain $\eta=-2.1 \%$ ), the bandgap increased, argued to be related to strain-induced polarization from the tetragonal distortion. On the other hand, for $\mathrm{KNO} / / \mathrm{STO}(\eta=-2.8 \%)$, the authors observed a decrease in structural symmetry from orthorhombic to tetragonal, with a concomitant rather large decrease in the bandgap. Such a result requires further study, to determine if it is a general effect for perovskite oxides or if it is confined to this particular system.

Liu et al. measured the optical bandgap and refractive index of strained BFO thin films grown on a range of substrates. ${ }^{128}$ Temperature-dependent ellipsometry evidenced a weak change in the bandgap with strain. Interestingly, the change in bandgap with temperature showed a slight change in slope at the Néel transition, which suggests spin-lattice coupling in these films. A subsequent ellipsometry and firstprinciples calculation study ${ }^{86}$ showed that epitaxial strain in BFO thin films induces a small change in the optical bandgap [Fig. 8(a)], but, more importantly, induces a large variation of the optical refractive index $n$. As mentioned in Sec. III D, this change is so large that it suggests that the elasto-optic coefficients of BFO are larger than those of the typical acousto-optic modulator media such as quartz or lithium niobate. Such a large elasto-optic response in BFO offers attractive perspectives for thin-film based surface acoustic wave (SAW) deflectors and modulators. Although not based on BFO, a few such device prototypes are described in Sec. V.

It is impossible to consider epitaxial strain (usually homogeneous or averaged over the film thickness) without mentioning inhomogeneous strain. This type of strain can induce very large flexoelectric fields ${ }^{129}$ through the strain gradients created-usually in thin films by the process of strain relaxation. To understand if strain gradients influence the optical bandgap of BFO, a large set of films grown on STO substrates was characterized by XRD and transmittance measurements. ${ }^{106}$ It was found that besides the usual dispersion of the data, the bandgap showed no systematic trend on 
(a)

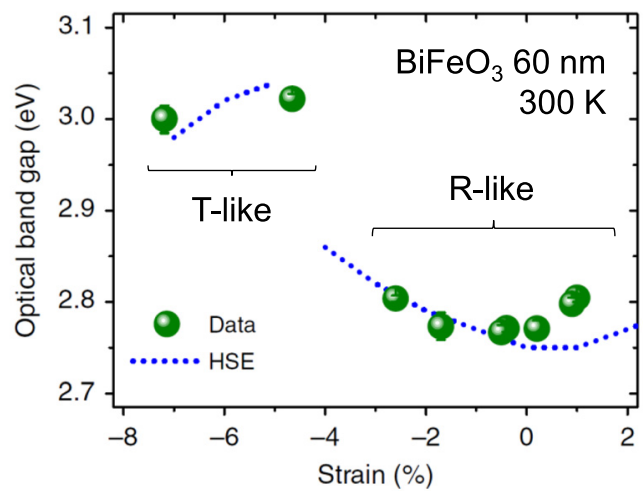

(c)

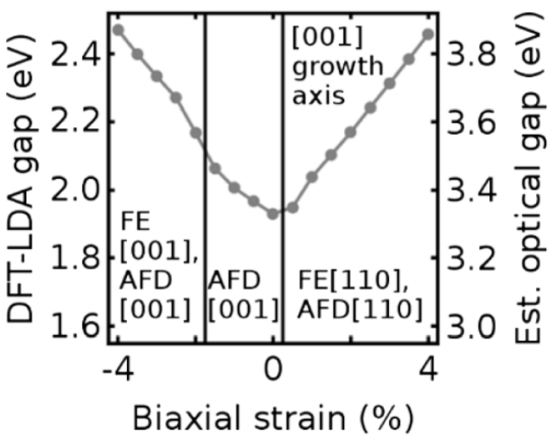

(b)

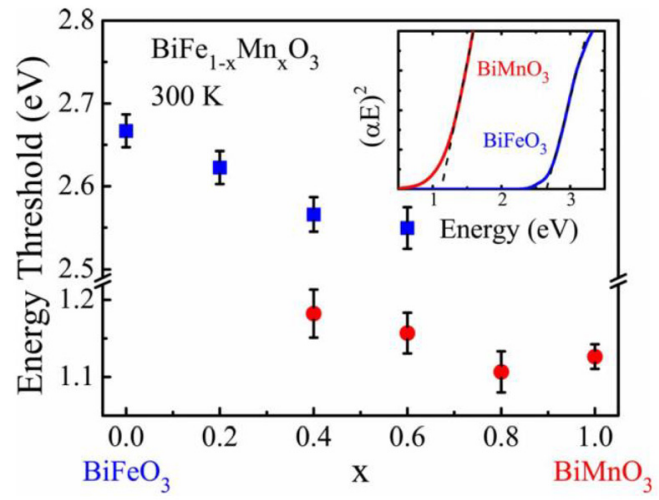

(d)

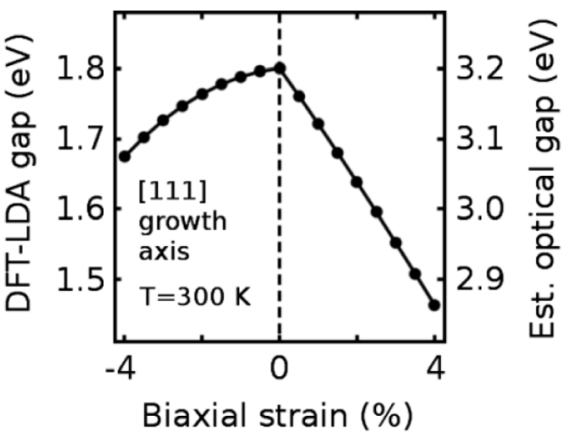

FIG. 8. Bandgap tuning in $\mathrm{ABO}_{3}$ perovskite thin films. (a) Epitaxial strain changes the bandgap in the $R$-like phase of BFO, albeit in the upwards direction, while the $T$-like phase has a larger bandgap of $\sim 3.0 \mathrm{eV}$ (from Ref. 86). (b) Using a solid solution of BFO-BMO to reduce bandgap. Reproduced with permission from Appl. Phys. Lett. 96, 192901 (2010). Copyright 2010 AIP Publishing LLC. (c) $\mathrm{SrTiO}_{3}$ films in the (001) orientation are predicted to have an upward shift of the bandgap for both compressive and tensile strains; (d) the converse trend is predicted for (111) epitaxy of $\mathrm{SrTiO}_{3}$ films. Reproduced with permission from R. F. Berger et al., Phys. Rev. Lett. 107, 146804 (2011). Copyright 2011 American Physical Society.

the inhomogeneous strain — a somewhat surprising result since one may expect oxygen vacancies to accumulate at the misfit dislocations which are in high concentrations in films with large strain gradients. ${ }^{129}$

The influence of strain on the electro-optic effect in complex oxide thin films is not well understood. One report showed that the EO response of films grown on different substrates was different, ${ }^{11}$ but it was not clear that the epitaxial strain was the dominant effect, as other influences such as changes in stoichiometry may have had an influence. Given the considerable strain-sensitivity of the FE polarization in $\mathrm{BTO},{ }^{130}$ and the intimate link between polarization and EO coefficients, ${ }^{131}$ it is probable that the EO response of $\mathrm{ABO}_{3}$ perovskites will be sensitive to epitaxial strain. Further work, using a wider range of fully strained films, is required to elucidate the influence of strain on the $\mathrm{EO}$ response.

In summary, the role of strain on the optical properties of FE oxide films still remains relatively uncharted territory. Indeed, many avenues have yet to be explored, including using for instance the strain-induced changes in band structure of perovskites to tune the bandgap of Mott insulators (whose gaps are rather low and could thus be attractive for solar energy harvesting). ${ }^{132}$ Modifying the electro-optic response by strain is another promising approach to obtain giant responses from multiferroics or ferroelectrics. As we have seen, an important influence of the imposed strain is on the band structure of the FE material. Such changes therefore influence the bandgap and particularly the optical absorption. We consider this in Sec. III F.

\section{F. Bandgap engineering}

The ultimate aim with strain tuning of FE oxides is to have a means to control the bandgap on demand. While in the case of EO devices, transparency for the optical beam in the medium is a requisite (thus needing a higher bandgap), the contrary (i.e., better absorption) is required for photovoltaic applications. Most ferroelectric oxides have bandgaps well above the visible range (e.g., 3-4 eV), which, in principle, makes them good candidates for transparency in the visible and IR (e.g., at $\lambda=1500 \mathrm{~nm}$ ) (see Sec. V for more detail about waveguide requirements), but their intrinsic energy harvesting capabilities are rather low. Significant efforts to reduce these values have been made. We consider such efforts in the following, which serves as an introduction to Sec. IV devoted to the bulk photovoltaic effect.

The influence of epitaxial strain on the optical bandgap of $\mathrm{SrTiO}_{3}$ was explored theoretically by Berger et al. ${ }^{125} \mathrm{It}$ was found that in (001) oriented films, both compressive and tensile strains increase the bandgap [Fig. 8(c)], with $\sim 4 \%$ strain increasing the gap by about $0.5 \mathrm{eV}$. Unfortunately, this 
strain-induced modification of the gap is upwards, thus not helping for solar energy applications. Conversely, they predicted that for films grown in the (111)-orientation, biaxial strain can induce a decrease in the optical bandgap [Fig. $8(d)$ ]. While such a prediction is encouraging, difficulties in imposing strain in the (111) orientation is likely to be the limitation, as usually the films relax at a very low thickness thereby practically limit the amount of bandgap tunability.

Another theoretical work proposed that by using a polarization rotation strategy, the bandgap of FE oxides could be reduced by as much as $1.2 \mathrm{eV} .{ }^{133}$ Using KNO under epitaxial strain as a test case, the calculations suggest that $2 \%$ of strain can reduce the gap from $2.4 \mathrm{eV}$ down to $1.7 \mathrm{eV}$. Such a prediction has yet to be confirmed experimentally.

Several experimental attempts have been made to engineer the bandgap of epitaxial FE films. An intuitive approach is to use a solid-solution route whereby the ferroelectric is mixed with a low band-gap material. ${ }^{134}$ The critical point here is to ensure that the polar nature of the solution be retained so that the intrinsic charge separation mechanism remains intact. To demonstrate simple bandgap tunability, $\mathrm{Xu}$ et al. investigated the bandgap response of $\mathrm{Bi}\left(\mathrm{Fe}_{1-\mathrm{x}} \mathrm{Mn}_{\mathrm{x}}\right) \mathrm{O}_{3}$ epitaxial films and found that the response was roughly the linear superposition of the end members [Fig. 8(b)] ${ }^{135}$ As a result, the bandgap was reduced from 2.67 for $\mathrm{BFO}$ to $1.1 \mathrm{eV}$ for $\mathrm{BiMnO}_{3}$. That said, it is important to stress that $\mathrm{BMO}$ is probably not ferroelectric, ${ }^{136}$ unless strained appropriately. ${ }^{137}$ So it may be that although the bandgap is reduced dramatically, the charge separation mechanism may be lost in the process. As will be discussed later, one method to overcome this intrinsic limitation is using a multilayered approach.

It was shown by Chernova et al. that the bandgap of $\mathrm{BaTiO}_{3}$ can be tuned by thickness-dependent strain. ${ }^{138}$ BTO films of thickness between 8 and $21 \mathrm{~nm}$ were grown epitaxially on STO substrates, and the optical properties measured by spectroscopic ellipsometry. It was found that for the thinnest film, with an out-of-plane strain of $1.3 \%$, the bandgap was increased by $\sim 0.3 \mathrm{eV}$ relative to a bulk BTO crystal. The increase in bandgap was ascribed to a strain-dependent increase in the ferroelectric polarization and thus bandgap through the relation $\Delta E=\beta P^{2}$ with $\beta$ being the "polarization potential" of BTO (Ref. 139).

In conclusion, a ferroelectric material with a considerably reduced bandgap would absorb more of the solar spectrum and through the so-called bulk photovoltaic effect (BPV) thus generate significant photocurrents. Section IV describes the BPV effect and discusses its promise for energy harvesting.

\section{BULK PHOTOVOLTAIC EFFECT IN THIN FILM FERROELECTRICS}

Conventional $p-n$ junctions rely on thermalized photoexcited electrons (and holes) that have relaxed some of their energy through interactions with lattice vibrations. The excess energy carried by photons with above bandgap energy is lost during the thermalization process (on a time scale of $10^{-12}-10^{-13} \mathrm{~s}$ ), and all thermalized photo-excited electron- hole pairs have roughly the same energy $E_{\mathrm{g}}$, equal to the bandgap of the material. As a result of this relaxation process, a large amount of harvestable energy cannot be collected. This gives rise to the fundamental Shockley-Queisser limit, which fixes a thermodynamical upper limit for conversion efficiency, in any single cell relying on thermalized photo-excited carriers, to $26 \%$, with an optimal bandgap of about $1.1 \mathrm{eV}{ }^{140}$

On the other hand, any material lacking inversion symmetry - such as ferroelectrics - can potentially exhibit a bulk photovoltaic (BPV) effect. This effect relies on the momentum or real space shift asymmetry of the population of photogenerated carriers that have not yet been thermalized. ${ }^{141}$ Hence, the above bandgap part of the solar spectrum can, in principle, be fully used without loss of energy caused by thermalization processes, thus allowing the Shockley-Queisser limit to be overcome. The main obstacle is the extremely short lifetime of these so-called "hot" (i.e., non-thermalized) carriers, meaning that they must be efficiently extracted.

One method for achieving efficient carrier extraction is to use nanoscopic probes. For instance, using a $\mathrm{BiFeO}_{3}$ single crystal and an atomic force microscopy (AFM) tip as top electrode, an enhancement of the external quantum efficiency (or EQE, the ratio of the number of charge carriers collected by the number of incident photons) was reported. The observed enhancement was by a staggering seven orders of magnitude when compared to the more conventional design where a planar electrode covered the entire surface of the sample. ${ }^{142}$ A similar approach was used on $\mathrm{BaTiO}_{3}$ (BTO) monodomain single crystals, for which a power conversion efficiency (PCE) of $4.8 \%$ under AM1.5G illumination (the standard for the characterization of solar cells) was measured. This PCE is 50\% larger than the Shockley-Queisser limit for a material with similar bandgap relying on thermalized electrons to operate. ${ }^{143}$

It is important to realize that the BPV effect is fundamentally different from the physical principles of conventional solar cells. Typically, BPV-based solar cells can exhibit huge open-circuit photovoltages $V_{\mathrm{oc}}$ (the voltage measured in an illuminated sample under open-circuit conditions) that can drastically exceed the bandgap equivalent voltage $E_{\mathrm{g}} / e$. Note that in $p-n$ junctions $V_{\text {oc }}$ cannot exceed $E_{\mathrm{g}} / e$, due to the thermalization process, as explained above. Ferroelectrics thus exhibit attractive photovoltaic characteristics that can be exploited as alternatives to and/or in tandem with conventional semiconductor-based $p$ - $n$-based solar cells.

Observed for the first time in 1956 in BTO,${ }^{144}$ the BPV in ferroelectrics underwent a revival in the late 2000s, by virtue of the capability to grow high quality epitaxial thin films in a controlled manner. For example, in 2010, Yang et al. grew BFO thin films with well-ordered arrays of either $71^{\circ}$ or $109^{\circ}$ domain walls (DWs) on (110)-oriented $\mathrm{DyScO}_{3}$ substrates. ${ }^{89}$ Illuminating the sample with $28.5 \mathrm{~mW} / \mathrm{cm}^{2}$ white light with the Pt electrodes oriented parallel to the DWs, generated a photovoltage $V_{\mathrm{oc}}=16 \mathrm{~V}$ (i.e., larger than the bandgap), along with an in-plane photocurrent density $j_{s c}=120 \mu \mathrm{A} \mathrm{cm}^{-2}$ flowing against the polarization. On the other hand, when the electrodes were oriented perpendicular 
(a)

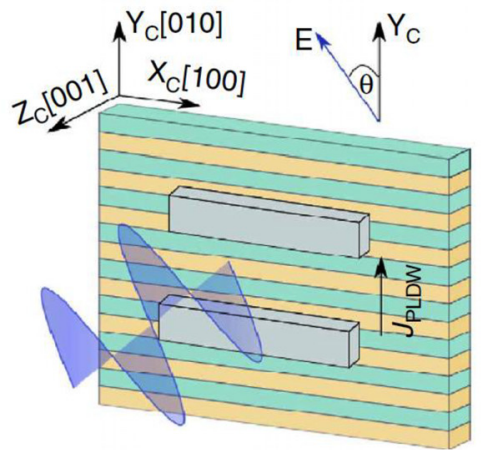

(b)

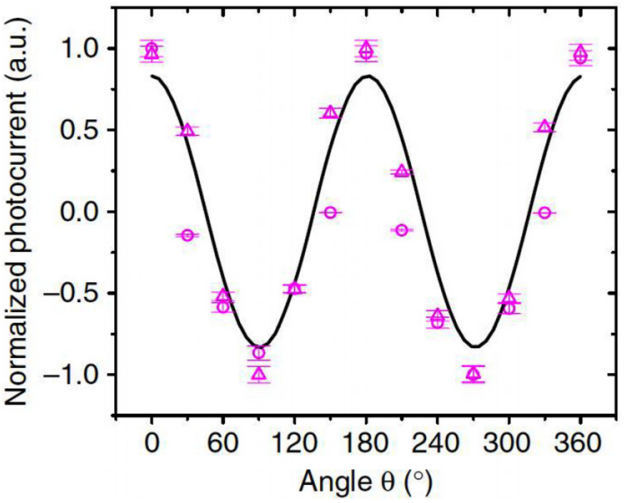

FIG. 9. Bulk photovoltaic effect in a $\mathrm{BiFeO}_{3}$ thin film shown to have no dependence on domain wall type. (a) Geometry of the experiment, where a light beam linearly polarized at angle $\theta$ induces a photocurrent, which is then collected with electrodes oriented parallel with the domain walls. (b) Dependence of the photocurrent on the angle $\theta$ for samples with $71^{\circ}$ and $109^{\circ}$ DWs shows the same behavior. Reproduced with permission from A. Bhatnagar et al., Nat. Commun. 4, 2835 (2013). Copyright 2013 Macmillan Publishers Limited. to the DWs, this photovoltaic effect was not observed. The proposed explanation used concepts not dissimilar to the space charge region in a $p-n$ junction: the domain wall width (of the order of 1-2 nm) was suggested to act like the depletion region, in which an internal electric field arising from the potential drop was efficiently acting to separate the photogenerated electrons and holes.

This explanation, however, was controversial, and it was shown later that the DW contribution in the photovoltaic effect in ferroelectrics is related only to conductivity. ${ }^{145}$ Indeed, several works report that DWs possess a higher conductivity than the domains they separate, e.g., in $\mathrm{BiFeO}_{3}$ (Refs. 146-148) and PZT. ${ }^{149}$ The origin of this enhanced conductivity is believed to be related to the release of free carriers from defects which tend to be preferentially localized at DWs. ${ }^{150,151}$ The photovoltaic effect observed in ferroelectrics thus has no clear direct link with DWs and is more likely due to the so-called bulk photovoltaic (BPV) effect inherent to any material lacking inversion symmetry, ${ }^{152}$ such as ferroelectrics.

From a phenomenological point of view, since the BPV effect is a second order nonlinear process, it is described by a third rank tensor. For a non-centrosymmetric material illuminated by monochromatic light with polarization vector $e$ and intensity $I$, the contribution to the current density $j$ flowing through the sample arising from the BPV effect ${ }^{153}$ is written

$$
j_{B P V}=\beta_{i j l} e_{j} e_{l}^{*} I
$$

where the BPV tensor $\beta_{i j l}$ is, in general, complex. It turns out that one of the specific response of the BPV effect is that if the sample is illuminated by linearly polarized light, the short-circuit photocurrent $j_{s c}$ oscillates as $\sin (2 \theta)$, with $\theta$ being the angle of the light polarization. Such a phenomenon was observed in BFO thin films with either $71^{\circ}$ or $109^{\circ} \mathrm{DWs}$ deposited on a $\mathrm{TbScO}_{3}$ substrate (see Fig. 9). ${ }^{145}$

Although the BPV effect is rather easy to understand from pure symmetry arguments, its microscopic origin can be split in two contributions: ballistic and shift current. 5,154 The ballistic contribution to the BPV effect arises from asymmetric transition rates in momentum space. This generates a net current due to a non-vanishing global momentum of the non-thermalized ("hot") photogenerated carriers (electrons and holes) which have, on average, a preferred momentum direction. Upon thermalization through scattering processes, the information on the direction of the carriers' momentum is lost, and they no longer contribute to the bulk photovoltaic current. This contribution to the photovoltaic current is thus coined "ballistic," and it depends critically on the mean free path of the excited electrons, on the order of 10 to $100 \mathrm{~nm} .{ }^{153}$ This mechanism actually corresponds to the pioneering BPV model developed by Glass ${ }^{155,156}$ where the short-circuit photovoltaic current $j_{s c}$ is written

$$
j_{B P V}^{s c} \propto \Lambda \alpha(\hbar \omega) \frac{I}{\hbar \omega}
$$

with $\alpha(\hbar \omega)$ being the absorbance of the sample at photon energy $\hbar \omega, I$ being the incident light power, and $\Lambda$ being the mean free path quantity (which is the weighted average between the shift displacement of the fraction of electrons excited into bound states, and the mean free path of photoexcited free electrons before electron-electron or electronphonon scatterings render their momentum isotropic). Thus, it was pointed out earlier that only the photogenerated carriers within a mean free path distance from the interface contribute significantly to the BPV photocurrent. Consequently, an enhancement of the PCE up to $1 \%$ in $20 \mathrm{~nm}$ thick BTO films is achieved when reducing the dimension between the electrodes of the photo-active element. ${ }^{157}$ Similarly, an increase in the photocurrent density from $0.25 \mu \mathrm{A} \mathrm{cm}^{-2}$ to $1.5 \mu \mathrm{A} \mathrm{cm}^{-2}$ was obtained when diminishing the thickness of a BFO film deposited on STO from $450 \mathrm{~nm}$ to below $100 \mathrm{~nm}$ with a $435 \mathrm{~nm}$ incident wavelength light with the intensity of $20 \mathrm{~mW} \mathrm{~cm}^{-2} .158$

Note that the ballistic contribution described by Eq. (11) does not have a polarization and tensorial dependence. These subtleties naturally appear when considering the shift current contribution to the BPV current. In addition to asymmetric transition rates in momentum space (ballistic current), the excitation of an electron (hole) can shift its average position in real space (shift current) and produce a net current. Recently, the shift current contribution to the BPV effect was implemented in first-principles calculations, ${ }^{159}$ which showed that the major photovoltaic contribution in BFO does not come from the photo-response of DWs (Ref. 160). To design photovoltaic (PV) efficient architectures to 
enhance the photocurrent output, it is possible for instance to engineer alternating nanolayers of $\mathrm{PbNiO}_{2}$ (a perovskite with an oxygen vacancy) and $\mathrm{PbTiO}_{3}$ to effectively lower the bandgap and tailor the PV output such that all absorbed photons (at any frequency) contribute constructively. ${ }^{161}$

As mentioned in Sec. III F, the obvious limitation to the BPV effect in FE materials is the rather high bandgap energy, typically above $3.2 \mathrm{eV}$. Since the maximum of the solar spectrum is at about $1.5 \mathrm{eV}$, various strategies for lowering the bandgap have been suggested. Among these, the simplest is to play with chemistry, by using solid solutions or doping. For instance, the combination of $\mathrm{Ni}^{2+}$ and oxygen vacancies in the solid solution $\left[\mathrm{KNbO}_{3}\right]_{1-\mathrm{x}}-\left[\mathrm{BaNi}_{1 / 2} \mathrm{Nb}_{1 / 2} \mathrm{O}_{3-\delta}\right]_{\mathrm{x}} \quad(\mathrm{KBNNO})$ was experimentally demonstrated to drastically reduce the bandgap from $3.8 \mathrm{eV}$ in the regular ferroelectric $\mathrm{KNbO}_{3}$ to $1.18 \mathrm{eV}$ in KBNNO, yielding a large short-circuit photocurrent $j_{s c}$ of $0.1 \mu \mathrm{A} \mathrm{cm}{ }^{-2}$ under $4 \mathrm{~mW} \mathrm{~cm}^{-2}$ halogen lamp illumination. ${ }^{134}$

One can also play with the structure and the chemical ordering to achieve an efficient reduction of the bandgap and improved PV performance. In perovskite structures with very high tetragonality, such as $\mathrm{Bi}(\mathrm{Zn}, \mathrm{Ti}) \mathrm{O}_{3}$ (BZT), the bandgap has been shown to be very sensitive to local chemical ordering of the B-cation. For alternate stacking of planes of $\mathrm{Zn}$ and Ti cations in the tetragonal direction, DFT calculations showed that the bandgap is decreased by about $0.5 \mathrm{eV}$ when compared to other chemically ordered cation arrangements. ${ }^{162}$ In fact, the bandgap of the tetragonal structure of various perovskites is systematically found to be lower than its rhombohedral counterpart ${ }^{133}$ (with the exception, it seems, of $\mathrm{BiFeO}_{3}$ - the reason for which is discussed in Ref. 86) which is interesting from an epitaxial thin film perspective, as the tetragonal structure is readily obtained in films through compressive strain. In such tetragonal structures, epitaxial strain can suppress the oxygen octahedra rotations, which also acts to lower the bandgap. ${ }^{162}$ In $\mathrm{Bi}_{2} \mathrm{FeCrO}_{6}$ (BCFO) epitaxial thin films, chemical ordering of $\mathrm{Cr}$ and $\mathrm{Fe}$ cations lowers the bandgap of pure BFO $(2.6 \mathrm{eV})$ down to $1.43 \mathrm{eV}$ through Jahn-Teller distortions. ${ }^{4}$ Controlling the chemical ordering by the deposition rate hence allowed to grow three multilayers of the same material, BFCO, with each layer absorbing a different part of the solar spectrum through their distinct chemical ordering. ${ }^{4}$ Such designed multilayered architectures to absorb a broader part of the solar spectrum yielded the highest PCE (8.1\%) ever recorded for a ferroelectric, with characteristic photovoltaic outputs $J_{\mathrm{sc}}=11.7 \mathrm{~mA} \mathrm{~cm}^{-2}$ and $V_{\mathrm{oc}}=0.79 \mathrm{~V}$ under AM1.5 illumination. Note that an earlier work on BCFO monolayer thin films by the same group showed a $6.5 \%$ PCE under a red laser and a photovoltaic current density of $0.99 \mathrm{~mA} \mathrm{~cm}^{-2}$, one of the largest ever recorded. ${ }^{163}$

It is also worth mentioning that ferroelectrics (and more generally, multiferroics), in addition to their BPV characteristics, offer unique features that go beyond solar cell applications. Recall that the BPV effect is described by a third rank tensor. Switching the polarization direction (which is the definition of a ferroelectric) is equivalent to reversing the inversion symmetry, and as a result the photovoltaic current should also be reversed. Therefore, concomitant switching of the photovoltaic short-circuit current and the ferroelectric polarization can be realized. In some cases, a perfect correspondence between the ferroelectric hysteresis and photocurrent loops has been demonstrated. ${ }^{164,165}$ For instance, in a $\mathrm{SrRuO}_{3} / \mathrm{BiFeO}_{3} / \mathrm{Pt}$ film stack under $10 \mathrm{~mW} \mathrm{~cm}^{-2}$ whitelight illumination, the photocurrent density of $0.1 \mu \mathrm{A} \mathrm{cm} \mathrm{cm}^{-2}$ was fully reversed upon switching the polarization between the UP and DOWN states. In contrast, in a mixed state comprising the same density of UP and DOWN domains, the photocurrent was shown to vanish. ${ }^{164}$

Similar poling-induced changes in the photovoltaic current direction, or at least tailoring of its magnitude upon switching, have been reported in BFO (Refs. 166-170), and in other ferroelectrics such as PLZT. ${ }^{165}$ In some cases, however, the Schottky barrier that exists at the metal-ferroelectric interface has resulted in cases of non-switching. ${ }^{167,169}$ This is due to the fact that the Schottky barrier can also be changed by the polarization which imposes a positive or negative surface charge layer at the interface, depending if the polarization is pointing downward or upward the interface, respectively. In (001)-oriented $\mathrm{BiFeO}_{3}$ films, the induced change in the barrier height (estimated to be $\pm 0.6 \mathrm{eV}$ ) was enough to change the nature of the Schottky barriers in the $\mathrm{Pt} / \mathrm{BiFeO}_{3} / \mathrm{SrRuO}_{3}$ structure from blocking to non-blocking diode effect and achieve switching of the forward direction of the ferroelectric diode and of the photovoltaic short-circuit current. ${ }^{164}$

The polarization directional dependence of the photocurrent has been used to implement a functional memory device. The photoferroelectric memory prototype comprised a $100 \mathrm{~nm}$ thick BFO thin film deposited on top of $\mathrm{La}_{0.7} \mathrm{Sr}_{0.3} \mathrm{MnO}_{3}$ with $\mathrm{Pt} / \mathrm{Fe}$ top electrodes. The binary information was encoded as the polarization direction, so writing was achieved by electrically switching the polarization, and the reading process involved measuring the photovoltaic current. ${ }^{168}$ It is interesting to note that the surface morphology of the FE film can play a significant role in the retention and stability of written domains when the film is illuminated by light, ${ }^{171}$ implying that when designing such photoferroelectrics memory devices, the materials processing and related film properties should be carefully controlled.

Finally, in addition to their switchability, ferroelectrics are good piezoelectrics. It has been observed that under illumination, ferroelectrics such as $\mathrm{BiFeO}_{3}$ deform through the photostriction effect. ${ }^{172,173}$ This effect is mostly considered to derive from the combined influences of the photovoltaic effect (which generates an electric field) and the converse piezoelectric effect (which translates this electric field into strain). ${ }^{173-176}$ This discovery opens pathways toward optically controlled transducer devices. In the case of multiferroics, one can also consider the magnetic degree of freedom: for instance, spin-polarized currents have been predicted to be generated directly from a BPV-like effect, ${ }^{177}$ thus extending the BFV effect to the field of spintronics. ${ }^{178}$ Coupling magnetization and BPV-photostriction has also been exploited to optically modify the magnetic coercivity of a $\mathrm{Ni}$ film grown on $\mathrm{B} \mathrm{BFeO}_{3}$ single crystal whose strain state was optically controlled through the photostriction effect. ${ }^{179}$

This section has described the unique photovoltaic properties that ferroelectric thin films can exhibit. There are 
many reasons to pursue the experimental and theoretical efforts in developing the BPV effect in ferroelectrics. The first is the tantalizing possibility of overcoming the Shockley-Queisser limit. The second is having the flexibility to be able to design nanostructures with optimal bandgap and thus engineer structures with a low mean-free path of the photogenerated carriers. Such efforts will be critical to the development of ferroelectric thin film nanostructures with improved PV performance.

\section{DEVICE PROTOTYPES}

Having described a range of the optical phenomena displayed by epitaxial FE thin films, we now present some examples of prototype EO modulators and other devices based on such heterostructures, keeping in mind that devices using the photovoltaic effects (i.e., solar cells) have already been described in Sec. IV. For a comprehensive review on EO modulator devices, the reader is directed to Ref. 3. To avoid repetition, here we focus on representative cases for each material family (just as was done for Sec. II) in describing particular prototype devices and some more recent results. We highlight a selection of such devices that use the electro-optic, acousto-optic effects, and include the design requirements for low-loss, high bandwidth integrated modulators. We begin by highlighting the importance of materials requirements for integrated modulators, by outlining some "design rules" for FE oxide thin films.

\section{A. Design rules for optical thin films for successful device integration}

Physical properties of substrate and film materials are summarized in Table II and Fig. 5. These data will assist the design of experiments and devices that use ferroelectric oxide thin films. For example, in many cases, lattice mismatch will influence the epitaxial growth of certain materials: e.g., growing $\mathrm{BaTiO}_{3}$ on $\mathrm{YAlO}_{3}$ will probably not work, due to the mismatch of more than $7 \%$.

Similarly, optical waveguiding in the thin film layer will not be possible if the refractive index of the film (which we denote $n_{\mathrm{f}}$ ) is lower than the refractive index of the substrate (denoted $n_{\mathrm{s}}$ ). This arises from the simple requirement to use total internal reflection (TIR) at the film-substrate interface to be able to confine light to the film layer. The light in the waveguide should be incident on the film-substrate interface at an angle larger than the critical angle $\theta_{c}$, given by $\sin \theta_{c}=n_{s} / n_{\mathrm{f}}$. Note that if $n_{\mathrm{f}}<n_{\mathrm{s}}$ then TIR cannot occur, which would be the case for a BST film on STO $\left(n_{\mathrm{BST}}=2.2\right.$; $\left.n_{\mathrm{STO}}=2.4\right)$. On the other hand, for R-BFO $\left(n_{\mathrm{BFO}}=2.9\right)$ on STO, the critical angle is found to be $\sim 55.85^{\circ}$. In this case, guided modes will be able to propagate in the film layer. A full discussion of guided waves in asymmetric waveguides is given in Sec. V B.

To determine the bandgap of a film (to, for instance, investigate thickness-dependent optical properties ${ }^{106}$ or some other strain-induced transition ${ }^{86}$ ) using standard optical transmittance measurements (where film thickness $\ll$ substrate thickness), the substrate bandgap should be higher than that of the film, so that the substrate absorption does not obscure that of the film (note that this is not a limitation if spectroscopic ellipsometry is used). So, for instance, using this method, one could determine bandgap of KNO $\left(E_{\mathrm{g}}=3.9 \mathrm{eV}\right)$ grown on $\mathrm{MgO}\left(E_{\mathrm{g}}=7.5 \mathrm{eV}\right)$, but not on STO $\left(E_{\mathrm{g}}=3.2 \mathrm{eV}\right)$. Finally, for operation at microwave frequencies-for instance, in EO modulators operating in the $\mathrm{GHz}$ range-then microwave phase matching is required. This is much more efficient if low dielectric constant substrates are used, as discussed in Refs. 44 and 180. The most attractive in this context is therefore MAS and $\mathrm{MgO}$. On the other hand, substrates popular for thin film growth such as STO will not be good choices from this point of view. It should be noted that in order to implement such modulators, appropriate buffering (e.g., with STO as demonstrated in Ref. 181) of the substrate would likely be required to promote epitaxial growth of the active layer. Similarly, optical waveguiding can be achieved using a substrate with a refractive index similar to the main film material, as long as a suitable buffer layer is used to separate the optical indices. ${ }^{15}$

\section{B. Electro-optic guided-wave modulator device proto- types based on FE thin films}

Various devices have been fabricated using EO thin films, such as Mach-Zehnder (M-Z) modulators, ${ }^{182,183}$ waveguide modulators, ${ }^{79}$ and beam deflectors. ${ }^{53}$ The general design of a so-called "strip-loaded" waveguide modulator is shown in Fig. 10(a).

To understand the design restrictions, let us consider an asymmetric planar waveguide structure similar to that in Fig. 10(a), where the substrate has refractive index $n_{\mathrm{s}}$, the film layer has refractive index $n_{\mathrm{f}}$, and the cover layer has refractive index $n_{\mathrm{c}}$. The cover layer may be air, in which case $n_{\mathrm{c}} \approx 1$. From guided wave optics theory, ${ }^{184}$ the number of independent parameters can be reduced and design rules can be established. (For full details, the reader is directed to Ref. 184.) First, we define the "effective guide index" given by $N=n_{\mathrm{f}} \sin \theta$, where $\theta$ is the angle of incidence in the waveguide (which is necessarily $>\theta_{\mathrm{c}}$, as explained above). We also define a dimensionless parameter $V$, the so-called "normalized frequency," as

$$
V=k t \sqrt{n_{\mathrm{f}}^{2}-n_{\mathrm{s}}^{2}}
$$

where $k=2 \pi / \lambda$ with $\lambda$ being the light wavelength and $t$ is film thickness. Finally, we define the "normalized guide index" $b$ and "asymmetry measure" $a$ [for transverse electric (TE) modes (the expression for TM modes is slightly different and can be found in Ref. 184)] as

$$
\begin{aligned}
& b=\frac{N^{2}-n_{s}^{2}}{n_{f}^{2}-n_{s}^{2}}, \\
& a=\frac{n_{s}^{2}-n_{c}^{2}}{n_{f}^{2}-n_{s}^{2}} .
\end{aligned}
$$

The value of $b$ is zero at cut-off (i.e., $\theta=\theta_{\mathrm{c}}$ ), while it approaches 1 for incidence angles much larger than the critical angle. The value of $a$ is zero for symmetric structures 


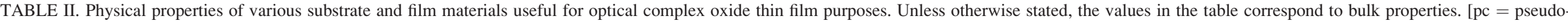

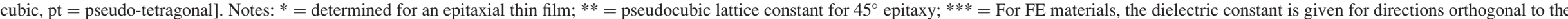
spontaneous polarization (cf. $\varepsilon_{a}$ in Table I). The dielectric constant in directions along polarization would in general be smaller than the values given here.

\begin{tabular}{|c|c|c|c|c|c|c|c|c|c|c|c|c|}
\hline Material & Abbreviation & $\begin{array}{l}\text { Crystal } \\
\text { structure }\end{array}$ & Family & $\begin{array}{l}\text { Lattice constants } \\
\text { (§) }\end{array}$ & $\begin{array}{l}\text { pc lattice } \\
(\AA)\end{array}$ & $\begin{array}{l}\text { Bandgap } \\
(\mathrm{eV})\end{array}$ & $n @ 633$ nm & $n @ 1550 \mathrm{~nm}$ & $n_{\mathrm{e}}-n_{\mathrm{o}} @ 633 \mathrm{~nm} n_{\mathrm{e}}$ & $\imath_{\mathrm{e}}-n_{\mathrm{o}} @ 1550 \mathrm{~nm}$ & $\varepsilon * * *$ & References \\
\hline \multicolumn{13}{|c|}{ (TYPICAL) SUBSTRATES } \\
\hline $\mathrm{MgO}$ & MGO & $\mathrm{NaCl}$ & Cubic & 4.216 & 4.216 & 7.5 & 1.735 & 1.715 & - & - & 10.2 & 57, 205, and 206 \\
\hline $\mathrm{MgAl}_{2} \mathrm{O}_{4}$ & MAS & FCC spinel & Cubic & 8.083 & 4.042 & 7.8 & 1.71 & 1.69 & - & - & 8.3 & 207-209 \\
\hline $\mathrm{PrScO}_{3}$ & PSO & Perovskite & Ortho & $5.780 ; 5.608 ; 8.025$ & 4.02 & $\sim 5.6$ & & & & & 30 & 210 and 211 \\
\hline $\mathrm{NdScO}_{3}$ & NSO & Perovskite & Ortho & $5.575 ; 5.776 ; 8.003$ & 4.008 & $5.52 *$ & & & & & 23 & 210 and 212 \\
\hline $\mathrm{KTaO}_{3}$ & KTO & Perovskite & Cubic & 3.989 & 3.989 & 3.55 & 2.21 & 2.12 & - & - & 209 & 127 and $212-215$ \\
\hline $\mathrm{SmScO}_{3}$ & SSO & Perovskite & Ortho & $5.527 ; 5.758 ; 7.965$ & 3.987 & $5.4-6$ & & & & & 18.5 & 210,212 , and 216 \\
\hline $\mathrm{GdScO}_{3}$ & GSO & Perovskite & Ortho & $5.480 ; 5.746 ; 7.932$ & 3.968 & $5.2-6.5$ & & & & & 20 & 212 and 216 \\
\hline $\mathrm{TbScO}_{3}$ & TSO & Perovskite & Ortho & $5.466 ; 5.731 ; 7.917$ & 3.96 & $5.6-6.1$ & & & & & 39.5 & 210 and 212 \\
\hline $\mathrm{DyScO}_{3}$ & DSO & Perovskite & Ortho & $5.440 ; 5.717 ; 7.903$ & 3.949 & $5.7-6.3$ & 2.06 & 2.03 & & & 21 & $208,210,212$, and 217 \\
\hline $\mathrm{SrTiO}_{3}$ & STO & Perovskite & Cubic & 3.905 & 3.905 & $3.2-3.4$ & 2.3 & 2.25 & - & - & 200 & 8, 57, 218, and 219 \\
\hline $\mathrm{Nb}: \mathrm{SrTiO}_{3}$ & $\mathrm{Nb}: \mathrm{STO}$ & Perovskite & Cubic & 3.905 & 3.905 & 3 & 2.42 & 2.27 & - & - & & 208 \\
\hline$(\mathrm{La}, \mathrm{Sr})(\mathrm{Al}, \mathrm{Ta}) \mathrm{O}_{3}$ & LSAT & Perovskite & Cubic & 3.868 & 3.868 & 5.5 & 2.02 & 1.98 & - & - & 23.7 & 208 and 220 \\
\hline $\mathrm{NdGaO}_{3}$ & NGO & Perovskite & Ortho & $5.426 ; 5.496 ; 7.707$ & 3.858 & 3.8 & & & & & 22.5 & 205 and 221 \\
\hline $\mathrm{SrLaGaO}_{4}$ & SLGO & Perovskite & Tetragonal & $3.84 ; 12.68$ & 3.84 & $>3.55$ & & & & & 21 & 205,222 , and 223 \\
\hline $\mathrm{Si}$ & $\mathrm{Si}$ & FCC diamond & Cubic & 5.431 & $3.84 * *$ & 1.14 & 3.88 & 3.67 & - & - & 11.6 & 224 and 225 \\
\hline $\mathrm{LaAlO}_{3}$ & LAO & Perovskite & Rhombo & 3.79 & 3.79 & 5.6 & 2.05 & 2.03 & & & 23.9 & 205,208 , and 226 \\
\hline $\mathrm{YAlO}_{3}$ & YAO & Perovskite & Ortho & $5.330 ; 7.375 ; 5.180$ & 3.702 & 8 & 1.96 & & & & 16 & 205 and $226-229$ \\
\hline $\mathrm{Y}: \mathrm{ZrO}_{2}$ & YSZ & Fluorite & Cubic & 5.12 & 3.62 & 4.96 & 2.152 & 2.11 & - & - & 27 & 230-232 \\
\hline $\mathrm{Al}_{2} \mathrm{O}_{3}$ & Saph & Corundum & Trigonal & $4.785 ; 12.991$ & N/A & $>6.2$ & 1.766 & 1.746 & -0.008 & -0.008 & 10.8 & 58,205 , and 233 \\
\hline \multicolumn{13}{|c|}{ (TYPICAL) FILMS } \\
\hline$(\mathrm{Pb}, \mathrm{La})(\mathrm{Zr}, \mathrm{Ti}) \mathrm{O}_{3}$ & PLZT & Perovskite & Rhombo/tetragonal & $4.04 ; 4.14$ & & $3.36-3.89$ & $2.41-2.67$ & & & & 600 & 234 and 235 \\
\hline $\mathrm{KNbO}_{3}$ & $\mathrm{KNO}$ & Perovskite & Ortho & $3.971 ; 4.016 ; 4.037$ & 4.008 & 3.95 & $2.17-2.33$ & $2.10-2.23$ & & & 160 & $28,32,33,127,207,236$, and 237 \\
\hline $\mathrm{BaTiO}_{3}$ & ВТО & Perovskite & Tetragonal & $3.99 ; 4.035$ & 4.005 & $3.2-3.3$ & 2.4 & 2.304 & -0.04 & -0.037 & 2300 & $8,138,207,218,238$, and 239 \\
\hline $\mathrm{PbTiO}_{3}$ & PTO & Perovskite & Tetragonal & $3.89 ; 4.14$ & 3.975 & 3.6 & 2.671 & & -0.01 & & 115 & $29-31$ and $240-242$ \\
\hline $\mathrm{R}-\mathrm{BiFeO}_{3}$ & $\mathrm{R}-\mathrm{BFO}$ & Perovskite & Rhombo/mono & $3.965(\mathrm{pc})$ & 3.965 & 2.75 & 3.05 & 2.7 & -0.24 & -0.18 & $30-50$ & $20,181,243$, and 244 \\
\hline$(\mathrm{Ba}, \mathrm{Sr}) \mathrm{TiO}_{3}$ & BST & Perovskite & Tetragonal & $3.905-3.99 ; 3.905-4.035$ & 3.954 & 3.5 & 2.2 & & & & 600 & 2 and 8 \\
\hline $\mathrm{NaNbO}_{3}$ & NNO & Perovskite & Ortho & $3.88 ; 3.902 ; 3.914$ & 3.899 & 3.78 & 2.24 & 2.12 & & & 750 & 236,245 , and 246 \\
\hline $\mathrm{GdTiO}_{3}$ & GTO & Perovskite & Ortho & $5.402 ; 5.697 ; 7.68$ & 3.895 & 0.7 & & & & & & 132 \\
\hline $\mathrm{T}-\mathrm{BiFeO}_{3}$ & T-BFO & Perovskite & Monoclinic & $3.75 ; 4.66(\mathrm{pt})$ & 3.75 & 3.05 & 2.76 & 2.5 & -0.259 & -0.242 & & $86,100,105$, and 247 \\
\hline $\mathrm{LiNbO}_{3}$ & LNO & Ilmenite & Trigonal & $5.149 ; 13.862$ & N/A & $3.6-3.7$ & 2.29 & 2.21 & -0.09 & -0.07 & 84 & $26-28,41,58,62,65,248-251$ \\
\hline $\mathrm{LiTaO}_{3}$ & LTO & Ilmenite & Trigonal & $5.153 ; 13.755$ & N/A & 3.93 & 2.183 & 2.12 & 0.006 & & 51 & $26,27,62,207,252$ \\
\hline
\end{tabular}


(a)

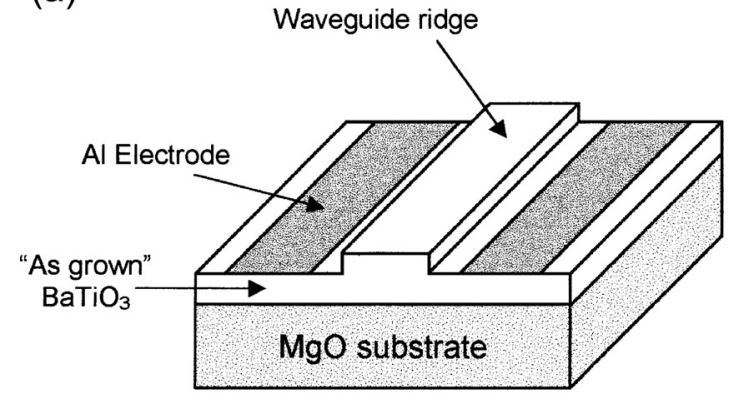

(b)

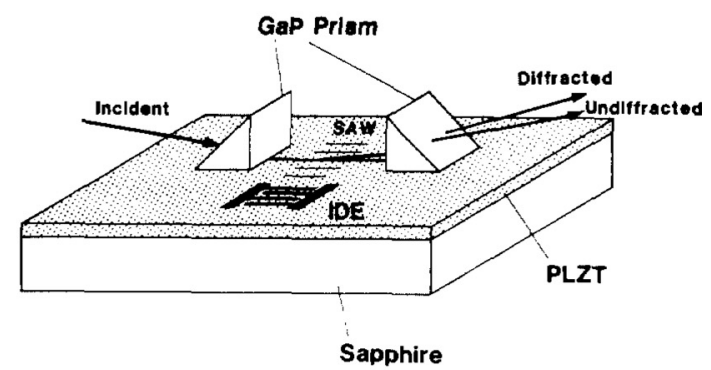

(c)
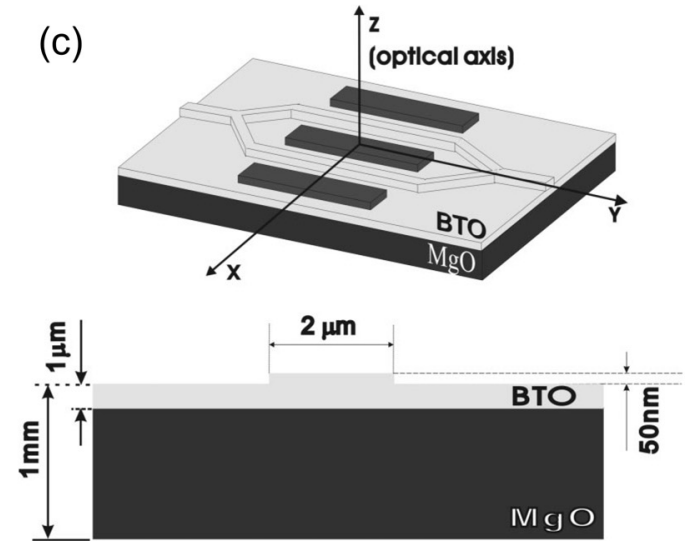

FIG. 10. Device prototypes based on ferroelectric oxide thin films. (a) General design of a strip-loaded waveguide modulator. Reproduced with permission from B. W. Wessels, J. Cryst. Growth 195, 706 (1998). Copyright 1998 Elsevier. (b) Acousto-optic deflector based on PLZT film on sapphire substrate. Reproduced with permission from H. Adachi and K. Wasa, IEEE Trans. Ultrason. Ferroelectr. Freq. Control 38, 645 (1991). Copyright 1991 IEEE. (c) MachZehnder modulator based on $\mathrm{BTO} / / \mathrm{MgO}$ as demonstrated by Petraru et al. Reproduced with permission from Appl. Phys. Lett. 81, 1375 (2002). Copyright 2002 AIP Publishing LLC.

$\left(n_{\mathrm{s}}=n_{\mathrm{c}}\right)$, while it can approach infinity for highly asymmetric structures. Following Ref. 184, the dispersion relation can be written in normalized form as

$$
V(1-b)^{\frac{1}{2}}=m \pi+\tan ^{-1}\left[\frac{b}{1-b}\right]^{\frac{1}{2}}+\tan ^{-1}\left[\frac{b+a}{1-b}\right]^{\frac{1}{2}},
$$

where $m$ is the mode number. A number of consequences arise from Eqs. (12)-(14). Putting $b=0$ and $m=0$ into Eq. (15) yields the normalized cutoff frequency $V_{0}$ for the fundamental mode

$$
V_{0}=\tan ^{-1} \sqrt{a} .
$$

A consequence of Eq. (16) is that for symmetric guides $(a=0)$ there is no lower limit to the film thickness $t$; that is, a fundamental mode always exists. For a strongly asymmetric guide $(a \rightarrow \infty)$, the cutoff is $V_{0}=\pi / 2$. This gives a lower limit to the film thickness for an asymmetric guide structure. This lower limit increases with decreasing filmsubstrate refractive index contrast. Finally, the cutoff $V_{m}$ for the $m$ th order mode is given by

$$
V_{m}=V_{0}+m \pi \text {. }
$$

This implies that the upper limit for the zeroth (fundamental) mode is $V_{1}=\tan ^{-1} \sqrt{a}+\pi$. In other words, to obtain single mode operation, the normalized frequency must fall within the following limits:

$$
\tan ^{-1} \sqrt{a}<V<\tan ^{-1} \sqrt{a}+\pi .
$$

As an illustrative example, we consider a $\mathrm{BiFeO}_{3}$ thin film $\left(n_{\mathrm{f}}=2.9\right)$ on STO substrate $\left(n_{\mathrm{s}}=2.4\right)$, with the cover layer being air $\left(n_{\mathrm{c}}=1\right)$. We use an operating wavelength of $\lambda=633 \mathrm{~nm}$. In this situation, the critical angle is $\theta_{\mathrm{c}}=55.85^{\circ}$, and the asymmetry measure from Eq. (14) is $a=1.80$. This asymmetry measure gives a lower and upper limit of the normalized frequency for the fundamental mode [from Eqs. (16) and (17)] of $0.930<V<4.072$. Substituting these parameters into Eq. (12), we obtain the following bounds on the film thickness for single-mode operation: $57.6 \mathrm{~nm}<t<252.0 \mathrm{~nm}$. We point out here that this simplistic analysis assumes perfect interfaces, completely homogeneous, and lossless films. While, in practice, the full waveguide structure should be modeled using finite element methods (such as done in Ref. 79), this simple example shows that for the typically obtainable refractive index contrasts between the film and the substrate of $0.1-0.4$ [cf. Figure 5(c)], single-mode operation can be achieved with film thicknesses of several hundreds of nanometers.

For a traveling-wave phase modulator operating at high frequencies, the microwave matching problem must be addressed. ${ }^{180}$ The phase velocity mismatch between the optical and microwave fields, which is determined by mostly by the dielectric constant of the substrate, ${ }^{8}$ sets the ultimate bandwidth of the device. These issues are further compounded by the fact that optical losses in thin films are almost always 
higher than high quality single crystals. Nonetheless, since the half-wave voltage $\left(V_{\pi}\right)$ in high-performance EO films can be orders of magnitude lower than, say $\mathrm{LiNbO}_{3}$ single crystals, the reduction in element size easily accounts for the increased losses, a fact which was recognized more than three decades ago. ${ }^{57}$ In the following, we discuss the progress on EO modulators and related technologies based on FE thin films grouped by the active FE used.

\section{Devices based on PLZT and related compounds}

In the 1980s, the focus was on PLZT; device prototypes such as waveguide switches, acousto-optic deflectors, and SAW EO deflectors were investigated. Wasa et al. ${ }^{185} \mathrm{dem}$ onstrated a total-internal reflection (TIR) waveguide switch using PLZT as the active medium as early as 1984. The device operated at $>1 \mathrm{GHz}$ speed and at a low voltage of 4.7 V. Other interesting applications of PLZT films were proposed, such as optical switches and SAW acousto-optic deflectors [Fig. 10(b)]. ${ }^{18}$ As early as 1993, the potential of using PLZT films for electrically written, optically read memories was recognized by Reitze et al. ${ }^{186}$

Early thin film EO devices began to be demonstrated in serious terms in the 1990s, mostly not only with BTO (to be discussed next) but also with PLZT. For example, Jin et al. demonstrated a Mach-Zehnder waveguide modulator using PLZT as the active element, with propagation losses of $2.7 \mathrm{~dB} / \mathrm{cm}$ and a half wave voltage of $8.5 \mathrm{~V} .{ }^{187}$ Masuda et al. ${ }^{183,188}$ demonstrated a Mach-Zehnder EO modulator with bandwidth up to $18 \mathrm{GHz}$, but performance up to $40 \mathrm{GHz}$ using PLZT epitaxial films on sapphire. The effective EO coefficients were up to $300 \mathrm{pm} / \mathrm{V}$ for low fields. There have also been recent efforts to incorporate PZT onto $\mathrm{Si}$ for optical applications. George et al. ${ }^{189}$ showed that using appropriate lanthanide based layers they were able to obtain preferentially oriented PZT films on silicon. The EO coefficients were rather strong at $110-240 \mathrm{pm} / \mathrm{V}$.

A PZT EO beam deflection device was reported by Nashimoto et al. ${ }^{53}$ In this work, the authors showed that PZT is an efficient layer for beam deflection. One limitation of the device was the unacceptable propagation loss of more than $17 \mathrm{~dB} / \mathrm{cm}$. It was suggested that instead of using a doped STO substrate, appropriate buffering could reduce the propagation loss.

\section{Barium titanate}

The 1990s and 2000s saw various groups focus on the design and optimization of BTO thin film EO modulators. ${ }^{3}$ One of the first prototypes in 1997 was based on BTO films grown epitaxially on $\mathrm{MgO} .{ }^{190}$ This modulator had modest effective EO coefficients of $r_{\text {eff }}=18 \mathrm{pm} / \mathrm{V}$ at $5 \mathrm{MHz}$. Four years later, Petraru et al.$^{79}$ demonstrated Mach-Zehnder modulators [Fig. 10(c)] in both the $a$ - and $c$-axis BTO orientations, once again, on $\mathrm{MgO}$ substrates. Propagation losses were $2-3 \mathrm{~dB} / \mathrm{cm}$, and the modulation performance was shown to be rather stable up to $1 \mathrm{MHz}$. Subsequent work focused on increasing the modulator bandwidth (up to $40 \mathrm{GHz}$ ), ${ }^{191}$ reducing the propagation losses (down to $\sim 1.1 \mathrm{~dB} / \mathrm{cm}$ ), ${ }^{192}$ and obtaining a lower voltage-length product (e.g., $0.5 \mathrm{~V} . \mathrm{cm}$ for
$5 \mathrm{~mm}$ long device at $\lambda=1561 \mathrm{~nm}) .{ }^{193}$ To reduce the phase velocity mismatch in such modulators, further work used strip-loaded waveguides (i.e., $\mathrm{Si}_{3} \mathrm{~N}_{4} / \mathrm{BaTiO}_{3} / \mathrm{MgO}$ ) incorporating photonic crystals. Using this approach, a half-wave voltage of only $V_{\pi}=5.5 \mathrm{~V}$ and a $3 \mathrm{~dB}$ bandwidth above $15 \mathrm{GHz}$ were demonstrated. ${ }^{194}$

An important technological step was made by Abel et al. ${ }^{195}$ when they incorporated BTO thin films grown by oxide MBE onto Si substrates by using a buffer layer of STO. Integration on silicon is very important for compatibility with current CMOS technology. ${ }^{196}$ Although the film was found to be polydomain, the authors were able to measure an effective EO coefficient $r_{\text {eff }}=148 \mathrm{pm} / \mathrm{V}$. This work highlights the requirement to control growth very carefully (particularly when integrating with $\mathrm{Si}$ ) and to properly characterize the EO response such that optimal devices can be fabricated. Further work from the same group shows that $\mathrm{BTO}$ on $\mathrm{Si}$ is a promising route for photonic switches, ring resonators, and EO modulators. ${ }^{197}$ Other work incorporating BTO on Si was reported by Xiong et al. ${ }^{198}$ Epitaxial BTO was directly grown on silicon-on-insulator (SOI), and the Pockels EO coefficient was found to be a large $213 \mathrm{pm} / \mathrm{V}$ in photonic modulators. In addition, bandwidth of a Mach-Zehnder modulator was shown to be $800 \mathrm{MHz}$, while for microring resonators it was as high as $4.9 \mathrm{GHz}$.

It has been shown theoretically that bandwidths up to $100 \mathrm{GHz}$ should be possible for BTO epitaxial thin film waveguides. As requirements for higher bandwidth increase, it may require a paradigm shift—e.g., away from bulk LNO modulators which are reaching their bandwidth limitstoward thin film integrated devices. ${ }^{199}$

\section{E. Bismuth ferrite}

The multiferroic compound bismuth ferrite is heavily studied, predominantly for its promise in electric-field controlled magnetism. ${ }^{200}$ In thin-film form, ${ }^{201}$ it has characteristics attractive for optical devices: low loss at $\lambda=1.55 \mu \mathrm{m},{ }^{181}$ bulk photovoltaic effect, and electro-optic coefficients on the same order as LNO. ${ }^{20}$ In particular, the integration of BFO onto low-dielectric constant substrates offers a voltage-length product which is extremely competitive with LNO. ${ }^{181}$ This is one avenue where BFO can in fact compete with the (generally) more attractive BTO. Using (001)-oriented BFO films grown on STO substrates grown by magnetron sputtering, Zhu et al. ${ }^{202}$ demonstrated a thin film electro-optic modulator. The effective EO coefficients were found to be around $19 \mathrm{pm} / \mathrm{V}$, in reasonable agreement with the previous characterization of $\mathrm{BFO}$ films. ${ }^{20}$ However, the losses of such a device were high at around $4 \mathrm{~dB} / \mathrm{cm}$, likely related to the multidomain nature of the film and the surface roughness. With more careful domain engineering, the losses could probably be reduced to the desired $1-2 \mathrm{~dB} / \mathrm{cm}$. Finally, BFO has been explored on a theoretical level for plasmonic waveguide modulators, ${ }^{203}$ but it remains to be seen how well such devices will perform in the experimental regime. 


\section{CONCLUSIONS}

This review has covered the history of epitaxial oxide thin films for optical applications. We began in the 1980 s when PLZT held a dominant position, and then progressed in the 1990s and 2000s to LNO and BTO-related materials. The past two decades showed BTO to be the stand-out FE oxide as BTO thin film integrated modulators could be successfully fabricated. From a fundamental perspective, it was shown that epitaxial strain plays a key role in tuning the optical response. Concurrently, the drive to discover new energy materials saw a renaissance in the use of ferroelectrics as photovoltaic materials, as well as for low power photonic applications. This has led to approaches such as bandgap tuning and substitutional engineering to achieve high performance ferroelectric-based solar cells.

The future of FE thin films for integrated optics will inevitably be linked intimately to the development photonics and optical computing technologies. This means that continuing improvements in thin film fabrication techniques and technology [e.g., PLD on large wafers ${ }^{204}$ for very large scale integration (VLSI)] are imperative to make FE thin film integrated optical devices part of the mainstream market. In addition, the added functionality of strain, superlattice, or compositionally engineered materials will be paramount. Materials such as multiferroics-where multiple ferroic orders coexist and may be coupled to one another-will offer new opportunities in hybrid devices that, for example, can respond not only to optical excitations but also to magnetic stimuli.

Some open questions still remain. How can one design large-scale manufactured thin-film integrated EO modulators that can compete with their bulk counterparts in terms of performance? Can we make complex oxide solar cells that can compete with $\mathrm{Si}$ or hybrid perovskites? Can disruptive technologies such as magnonics (the processing and transport of information by spin waves) be coupled with photonic functionalities, using for instance multiferroics such as BFO or magnetoelectric composites incorporating BTO? Can the carrier mobility issues be solved in FE oxide thin films to a point where such materials become commercially competitive? How far can predictive first-principles calculations take us toward such goals? Finally, returning back to EO modulators, can we implement domain engineering of films on an economical scale, on low dielectric constant substrates, in order to make highly efficient modulator devices?

These will be critical challenges that need to be addressed to transition the past four decades of exciting fundamental research progress in the optical properties of $\mathrm{FE}$ oxide thin films to mainstream commercial success.

\section{ACKNOWLEDGMENTS}

This research was partially supported by the Australian Research Council Centre of Excellence in Future Low-Energy Electronics Technologies (Project No. CE170100039) and funded by the Australian Government. Y.Y. thanks ONR Grant N00014-17-1-2818. C.P. acknowledges ARO Grant W911NF16-1-0227. L.B. thanks DARPA Grant HR0011-15-2-0038 (MATRIX program). B.D. would like to acknowledge a public grant overseen by the French National Research Agency (ANR) as a part of the "Investissements d'Avenir" program (Grant No. ANR-10-LABX-0035, Labex Nanoscalay).

The authors declare no competing interests.

${ }^{1}$ S. E. Miller, Bell Syst. Tech. J. 48, 2059 (1969).

${ }^{2}$ D.-Y. Wang, H. L. W. Chan, and C. L. Choy, Appl. Opt. 45, 1972 (2006).

${ }^{3}$ B. W. Wessels, Annu. Rev. Mater. Res. 37, 659 (2007).

${ }^{4}$ R. Nechache, C. Harnagea, S. Li, L. Cardenas, W. Huang, J. Chakrabartty, and F. Rosei, Nat. Photonics 9, 61 (2015).

${ }^{5}$ C. Paillard, X. Bai, I. C. Infante, M. Guennou, G. Geneste, M. Alexe, J. Kreisel, and B. Dkhil, Adv. Mater. 28, 5153 (2016).

${ }^{6}$ K. Preston, P. Dong, B. Schmidt, and M. Lipson, Appl. Phys. Lett. 92, 151104 (2008).

${ }^{7}$ a Petraru, J. Schubert, M. Schmid, O. Trithaveesak, and C. Buchal, Opt. Lett. 28, 2527 (2003)

${ }^{8}$ B. W. Wessels, J. Electroceram. 13, 135 (2004).

${ }^{9}$ M. E. Lines and A. M. Glass, Principles and Applications of Ferroelectrics and Related Materials (Oxford University Press, 1977).

${ }^{10}$ H. Adachi, T. Kawaguchi, and K. Setsune, Appl. Phys. Lett. 42, 867 (1983).

${ }^{11}$ D. Y. Wang, J. Wang, H. L. W. Chan, and C. L. Choy, J. Appl. Phys. 101, 043515 (2007).

${ }^{12}$ A. Boudrioua, J. C. Loulergue, E. Dogheche, and D. Remiens, J. Appl. Phys. 85, 1780 (1999).

${ }^{13}$ B. G. Potter, M. B. Sinclair, and D. Dimos, Appl. Phys. Lett. 63, 2180 (1993).

${ }^{14}$ T. Kang, B. Xiao, V. Avrutin, Ü. Özgür, H. Morkoç, J. W. Park, H. S. Lee, H. Lee, X. Wang, and D. J. Smith, J. Appl. Phys. 104, 093103 (2008).

${ }^{15}$ K. N. Nashimoto, S. N. Nakamura, T. M. Morikawa, and H. M. Moriyama, Jpn. J. Appl. Phys., Part 1 38, 5641 (1999).

${ }^{16} \mathrm{G}$. Yi, Z. Wu, and M. Sayer, J. Appl. Phys. 64, 2717 (1988).

${ }^{17}$ H. Adachi, T. Mitsuyu, O. Yamazaki, and K. Wasa, J. Appl. Phys. 60, 736 (1986).

${ }^{18} \mathrm{H}$. Adachi and K. Wasa, IEEE Trans. Ultrason. Ferroelectr. Freq. Control 38, 645 (1991).

${ }^{19}$ J. A. van Raalte, J. Opt. Soc. Am. 57, 671 (1967).

${ }^{20}$ D. Sando, P. Hermet, J. Allibe, J. Bourderionnet, S. Fusil, C. Carrétéro, E. Jacquet, J.-C. Mage, D. Dolfi, A. Barthélémy, P. Ghosez, and M. Bibes, Phys. Rev. B 89, 195106 (2014).

${ }^{21}$ E. L. Wooten, K. M. Kissa, A. Yi-Yan, E. J. Murphy, D. A. Lafaw, P. F. Hallemeier, D. Maack, D. V. Attanasio, D. J. Fritz, G. J. McBrien, and D. E. Bossi, IEEE J. Sel. Top. Quantum Electron. 6, 69 (2000).

${ }^{22}$ K. Buse, A. Adibi, and D. Psaltis, Nature 393, 665 (1998).

${ }^{23}$ D. Sando, E. Jaatinen, and F. Devaux, Appl. Opt. 48, 4676 (2009).

${ }^{24}$ D. Sando and E. Jaatinen, Opt. Express 21, 19510 (2013).

${ }^{25}$ I. M. Solskii, D. Y. Sugak, and M. M. Vakiv, Acta Phys. Pol. A 124, 314 (2013).

${ }^{26}$ A. W. Warner, M. Onoe, and G. A. Coquin, J. Acoust. Soc. Am. 42, 1223 (1967).

${ }^{27}$ S. H. Wemple, M. DiDomenico, and I. Camlibel, Appl. Phys. Lett. 12, 209 (1968).

${ }^{28}$ E. C. Subbarao, Ferroelectrics 5, 267 (1973).

${ }^{29}$ G. Shirane, S. Hoshino, and K. Suzuki, Phys. Rev. 80, 1105 (1950).

${ }^{30}$ V. G. Bhide, M. S. Hegde, and K. G. Deshmukh, J. Am. Chem. Soc. 51, 565 (1968).

${ }^{31}$ Z. Li, M. Grimsditch, X. Xu, and S.-K. Chan, Ferroelectrics 141, 313 (1993).

${ }^{32}$ A. K. Batra and M. D. Aggarwal, Pyroelectric Materials (SPIE, Washington, 2013).

${ }^{33}$ E. Wiesendanger, Ferroelectrics 6, 263 (1973).

${ }^{34}$ S. Triebwasser, Phys. Rev. 114, 63 (1959).

${ }^{35}$ D. Lebeugle, D. Colson, A. Forget, M. Viret, P. Bonville, J. F. Marucco, and S. Fusil, Phys. Rev. B 76, 024116 (2007).

${ }^{36}$ G. Catalan and J. F. Scott, Adv. Mater. 21, 2463 (2009).

${ }^{37}$ B. W. Wessels, M. J. Nystrom, J. Chen, D. Studebaker, and T. J. Marks, Mater. Res. Soc. Symp. Proc. 401, 211 (1996).

${ }^{38}$ L. W. Martin, Y. H. Chu, and R. Ramesh, Mater. Sci. Eng. R 68, 89 (2010).

${ }^{39}$ G. H. Haertling, Ferroelectrics 75, 25 (1987).

${ }^{40}$ W. E. Henderson, W. Laws Calley, A. G. Carver, H. Chen, and W. Alan Doolittle, J. Cryst. Growth 324, 134 (2011). 
${ }^{41}$ M. P. Sumets, V. A. Dybov, and V. M. Ievlev, Inorg. Mater. 53, 1361 (2017).

${ }^{42}$ V. Vaithyanathan, J. Lettieri, W. Tian, A. Sharan, A. Vasudevarao, Y. L. Li, A. Kochhar, H. Ma, J. Levy, P. Zschack, J. C. Woicik, L. Q. Chen, V. Gopalan, and D. G. Schlom, J. Appl. Phys. 100, 024108 (2006).

${ }^{43}$ D. M. Gill, B. A. Block, C. W. Conrad, B. W. Wessels, and S. T. Ho, Appl. Phys. Lett. 69, 2968 (1996).

${ }^{44}$ B. W. Wessels, J. Cryst. Growth 195, 706 (1998).

${ }^{45}$ D. K. Fork, F. Armani-Leplingard, and J. J. Kingston, Mater. Res. Soc. Symp. Proc. 361, 1993 (1995).

${ }^{46}$ F. J. Walker, R. A. McKee, H. W. Yen, and D. E. Zelmon, Appl. Phys. Lett. 65, 1495 (1994).

${ }^{47}$ S. H. Lee, T. W. Noh, and J. H. Lee, Appl. Phys. Lett. 68, 472 (1996).

${ }^{48}$ J. Tauc, Mater. Res. Bull. 3, 37 (1968).

${ }^{49}$ H. Jaffe and D. A. Berlincourt, Proc. IEEE 53, 1372 (1965).

${ }^{50}$ G. Teowee, J. M. Boulton, and D. R. Uhlmann, Integr. Ferroelectr. 20, 39 (1998).

${ }^{51}$ T. Kawaguchi, H. Adachi, K. Setsune, O. Yamazaki, and K. Wasa, Appl. Opt. 23, 2187 (1984)

${ }^{52}$ E. Dogheche, B. Jaber, and D. Rémiens, Appl. Opt. 37, 4245 (1998).

${ }^{53}$ K. Nashimoto, S. Nakamura, H. Moriyama, M. Watanabe, and E. Osakabe, Appl. Phys. Lett. 73, 303 (1998).

${ }^{54}$ K. Kurihara, M. Kondo, K. Sato, M. Ishii, N. Wakiya, and K. Shinozaki, Jpn. J. Appl. Phys., Part 1 46, 6929 (2007).

${ }^{55}$ S. Masuda, A. Seki, and Y. Masuda, Appl. Phys. Lett. 96, 072901 (2010).

${ }^{56}$ H. Adachi, T. Mitsuyu, K. Wasa, N. Suzuki, K. Tada, T. F. Properties, T. Kubota, and N. Tanaka, Jpn. J. Appl. Phys., Part 1 22, 11 (1983).

${ }^{57}$ M. Okuyama, T. Usuki, Y. Hamakawa, and T. Nakagawa, Appl. Phys. 21, 339 (1980).

${ }^{58}$ R. A. Betts and C. W. Pitt, Electron. Lett. 21, 960 (1985).

${ }^{59}$ A. Baudrant, H. Vial, and J. Daval, J. Cryst. Growth 43, 197 (1978).

${ }^{60}$ S. Miyazawa, S. Fushimi, and S. Kondo, Appl. Phys. Lett. 26, 8 (1975).

${ }^{61}$ S. Y. Lee, R. K. Route, and R. S. Feigelson, Mater. Res. Soc. Proc. 392, 177 (1995)

${ }^{62}$ Y. Sakashita and H. Segawa, J. Appl. Phys. 77, 5995 (1995).

${ }^{63}$ D. Kim, S.-M. Oh, S. Lee, and T. W. Noh, Jpn. J. Appl. Phys., Part 1 37, 2016 (1998).

${ }^{64}$ G. Balestrino, S. Martellucci, P. G. Medaglia, A. Paoletti, G. Petrocelli, A. Tebano, A. Tucciarone, F. Gelli, E. Giorgetti, S. Sottini, and L. Tapfer, Appl. Phys. Lett. 78, 1204 (2001).

${ }^{65}$ A. M. Marsh, S. D. Harkness, F. Qian, and R. K. Singh, Appl. Phys. Lett. 62, 952 (1993)

${ }^{66}$ K. Nashimoto, H. Moriyama, and E. Osakabe, Jpn. J. Appl. Phys., Part 1 35, 4936 (1996).

${ }^{67}$ E.-H. Dogheche, X. Lansiaux, and D. Remiens, Integr. Ferroelectr. 25, 47 (1999).

${ }^{68}$ N. Fujimura, M. Kakinoki, H. Tsuboi, and T. Ito, J. Appl. Phys. 75, 2169 (1994).

${ }^{69}$ Y. Kakehi, A. Okamoto, Y. Sakurai, Y. Nishikawa, T. Yotsuya, and S. Ogawa, Appl. Surf. Sci. 169-170, 560 (2001).

${ }^{70}$ Y. Shibata, K. Kaya, K. Akashi, M. Kanai, T. Kawai, and S. Kawai, Appl. Phys. Lett. 61, 1000 (1992).

${ }^{71}$ S. Fukunishi, Appl. Phys. Lett. 24, 424 (1974).

${ }^{72}$ C. H.-J. Huang, Integr. Ferroelectr. 6, 355 (1995).

${ }^{73}$ C. Buchal and M. Siegert, Integr. Ferroelectr. 35, 1731 (2001).

${ }^{74}$ G.-C. Yi, B. A. Block, and B. W. Wessels, Appl. Phys. Lett. 71, 327 (1997).

${ }^{75}$ H. S. Wang, K. Ma, D. F. Cui, Z. Q. Peng, Y. L. Zhou, H. B. Lu, Z. H. Chen, L. Li, and G. Z. Yang, J. Cryst. Growth 177, 67 (1997).

${ }^{76}$ L. Beckers, W. Zander, J. Schubert, P. Leinenbach, C. Buchal, D. Fluck, and P. Günter, Mater. Res. Soc. Proc. 441, 549 (1997).

${ }^{77}$ L. Beckers, J. Schubert, W. Zander, J. Ziesmann, A. Eckau, P. Leinenbach, and C. Buchal, J. Appl. Phys. 83, 3305 (1998).

${ }^{78}$ D. Kim and H. Kwok, Appl. Phys. Lett. 67, 1803 (1995).

${ }^{79}$ A. Petraru, J. Schubert, M. Schmid, and C. Buchal, Appl. Phys. Lett. 81, 1375 (2002)

${ }^{80}$ A. Rajamani, G. F. Dionne, D. Bono, and C. A. Ross, J. Appl. Phys. 98, 063907 (2005)

${ }^{81}$ J. Li, F. Duewer, C. Gao, H. Chang, X. D. Xiang, and Y. Lu, Appl. Phys. Lett. 76, 769 (2000).

${ }^{82}$ A. Y. Wu, F. Wang, C. Juang, C. Bustamante, C. Yeh, and J. Diels, in IEEE 7th International Symposium on Applications of Ferroelectrics (1990), pp. 135-138.

${ }^{83}$ D. Y. Kim, S. E. Moon, E. K. Kim, S. J. Lee, J. J. Choi, and H. E. Kim, Appl. Phys. Lett. 82, 1455 (2003).
${ }^{84}$ D. Lebeugle, D. Colson, A. Forget, and M. Viret, Appl. Phys. Lett. 91, 022907 (2007).

${ }^{85}$ D. Sando, A. Agbelele, D. Rahmedov, J. Liu, P. Rovillain, C. Toulouse, I. C. Infante, A. P. Pyatakov, S. Fusil, E. Jacquet, C. Carrétéro, C. Deranlot, S. Lisenkov, D. Wang, J.-M. M. Le Breton, M. Cazayous, A. Sacuto, J. Juraszek, A. K. Zvezdin, L. Bellaiche, B. Dkhil, A. Barthélémy, and M. Bibes, Nat. Mater. 12, 641 (2013).

${ }^{86}$ D. Sando, Y. Yang, E. Bousquet, C. Carrétéro, V. Garcia, S. Fusil, D. Dolfi, A. Barthélémy, P. Ghosez, L. Bellaiche, and M. Bibes, Nat. Commun. 7, 10718 (2016)

${ }^{87}$ J. Wang, J. Neaton, H. Zheng, V. Nagarajan, S. Ogale, B. Liu, D. Viehland, V. Vaithyanathan, D. Schlom, U. Waghmare, N. Spaldin, K. Rabe, M. Wuttig, and R. Ramesh, Science 299, 1719 (2003).

${ }^{88}$ T. Choi, S. Lee, Y. J. Choi, V. Kiryukhin, and S.-W. Cheong, Science 324, 63 (2009)

${ }^{89}$ S. Y. Yang, J. Seidel, S. J. Byrnes, P. Shafer, C. H. Yang, M. D. Rossell, P. Yu, Y. H. Chu, J. F. Scott, J. W. Ager, L. W. Martin, and R. Ramesh, Nat. Nanotechnol. 5, 143 (2010).

${ }^{90}$ Y. Lu, G.-H. Jin, M. Cronin-Golomb, S.-W. Liu, H. Jiang, F.-L. Wang, J. Zhao, S.-Q. Wang, and A. J. Drehman, Appl. Phys. Lett. 72, 2927 (1998).

${ }^{91}$ B. H. Hoerman, B. M. Nichols, and B. W. Wessels, Opt. Commun. 219, 377 (2003).

${ }^{92}$ P. Tayebati, D. Trivedi, and M. Tabat, Appl. Phys. Lett. 69, 1023 (1996).

${ }^{93}$ Y. Lu and R. J. Knize, J. Phys. D 37, 2432 (2004).

${ }^{94}$ D. G. Schlom, L.-Q. Chen, C. J. Fennie, V. Gopalan, D. A. Muller, X. Pan, R. Ramesh, and R. Uecker, MRS Bull. 39, 118 (2014).

${ }^{95}$ A. R. Akbarzadeh, L. Bellaiche, J. Íñiguez, and D. Vanderbilt, Appl. Phys. Lett. 90, 242918 (2007).

${ }^{96}$ M. Tyunina, J. Narkilahti, M. Plekh, R. Oja, R. M. Nieminen, A. Dejneka, and V. Trepakov, Phys. Rev. Lett. 104, 227601 (2010).

${ }^{97}$ J. H. Haeni, P. Irvin, W. Chang, R. Uecker, P. Reiche, Y. L. Li, S. Choudhury, W. Tian, M. E. Hawley, B. Craigo, A. K. Tagantsev, X. Q. Pan, S. K. Streiffer, L. Q. Chen, S. W. Kirchoefer, J. Levy, and D. G. Schlom, Nature 430, 758 (2004).

${ }^{98}$ I. C. Infante, S. Lisenkov, B. Dupé, M. Bibes, S. Fusil, E. Jacquet, G. Geneste, S. Petit, A. Courtial, J. Juraszek, L. Bellaiche, A. Barthélémy, and B. Dkhil, Phys. Rev. Lett. 105, 057601 (2010).

${ }^{99}$ F. Y. Bruno, K. Z. Rushchanskii, S. Valencia, Y. Dumont, C. Carrétéro, E. Jacquet, R. Abrudan, S. Blügel, M. Ležaić, M. Bibes, and A. Barthélémy, Phys. Rev. B 88, 195108 (2013).

${ }^{100}$ H. Béa, B. Dupé, S. Fusil, R. Mattana, E. Jacquet, B. Warot-Fonrose, F. Wilhelm, A. Rogalev, S. Petit, V. Cros, A. Anane, F. Petroff, K. Bouzehouane, G. Geneste, B. Dkhil, S. Lisenkov, I. Ponomareva, L. Bellaiche, M. Bibes, and A. Barthélémy, Phys. Rev. Lett. 102, 217603 (2009).

${ }^{101}$ D. Sando, B. Xu, L. Bellaiche, and V. Nagarajan, Appl. Phys. Rev. 3, 011106 (2016).

${ }^{102}$ J. Perdew, A. Ruzsinszky, G. Csonka, O. Vydrov, G. Scuseria, L. Constantin, X. Zhou, and K. Burke, Phys. Rev. Lett. 100, 136406 (2008).

${ }^{103}$ A. V. Krukau, O. A. Vydrov, A. F. Izmaylov, and G. E. Scuseria, J. Chem. Phys. 125, 224106 (2006).

${ }^{104}$ M. Gajdoš, K. Hummer, G. Kresse, J. Furthmüller, and F. Bechstedt, Phys. Rev. B 73, 045112 (2006).

${ }^{105}$ P. Chen, N. J. Podraza, X. S. Xu, A. Melville, E. Vlahos, V. Gopalan, R. Ramesh, D. G. Schlom, and J. L. Musfeldt, Appl. Phys. Lett. 96, 131907 (2010).

${ }^{106}$ D. Sando, C. Carrétéro, M. N. Grisolia, A. Barthélémy, V. Nagarajan, and M. Bibes, Adv. Opt. Mater. 6, 1700836 (2017).

${ }^{107}$ S. Ju and T.-Y. Cai, Appl. Phys. Lett. 95, 231906 (2009).

${ }^{108}$ J. F. Ihlefeld, N. J. Podraza, Z. K. Liu, R. C. Rai, X. Xu, T. Heeg, Y. B. Chen, J. Li, R. W. Collins, J. L. Musfeldt, X. Q. Pan, J. Schubert, R. Ramesh, and D. G. Schlom, Appl. Phys. Lett. 92, 142908 (2008).

${ }^{109}$ I. C. C. Chang, IEEE Trans. Sonics Ultrason. 23, 2 (1976).

${ }^{110}$ Y. Yang, C. Paillard, B. Xu, and L. Bellaiche, J. Phys: Condens. Matter 30, 073001 (2018).

${ }^{111}$ M. Bass, C. DeCusatis, and J. Enoch, Handbook of Optics, 2nd ed. (McGraw-Hill, New York, 2001).

${ }^{112}$ L. Chen, Y. Yang, Z. Gui, D. Sando, M. Bibes, X. K. Meng, and L. Bellaiche, Phys. Rev. Lett. 115, 267602 (2015).

${ }^{113}$ G. Catalan, A. Janssens, G. Rispens, S. Csiszar, O. Seeck, G. Rijnders, D. H. A. Blank, and B. Noheda, Phys. Rev. Lett. 96, 127602 (2006).

${ }^{114}$ G. Catalan, A. Lubk, A. H. G. Vlooswijk, E. Snoeck, C. Magen, A. Janssens, G. Rispens, G. Rijnders, D. H. A. Blank, and B. Noheda, Nat. Mater. 10, 963 (2011). 
${ }^{115}$ R. J. Zeches, M. D. Rossell, J. X. Zhang, A. J. Hatt, Q. He, C.-H. Yang, A. Kumar, C. H. Wang, A. Melville, C. Adamo, G. Sheng, Y.-H. Chu, J. F. Ihlefeld, R. Erni, C. Ederer, V. Gopalan, L. Q. Chen, D. G. Schlom, N. A. Spaldin, L. W. Martin, and R. Ramesh, Science 326, 977 (2009).

${ }^{116}$ Y. M. Jin, Y. U. Wang, A. G. Khachaturyan, J. F. Li, and D. Viehland, Phys. Rev. Lett. 91, 197601 (2003).

${ }^{117}$ M. Ahart, M. Somayazulu, R. E. Cohen, P. Ganesh, P. Dera, H. Mao, R. J. Hemley, Y. Ren, P. Liermann, and Z. Wu, Nature 451, 545 (2008).

${ }^{118}$ Z. Wu and R. E. Cohen, Phys. Rev. Lett. 95, 037601 (2005).

${ }^{119}$ A. Dejneka, D. Chvostova, O. Pacherova, T. Kocourek, M. Jelinek, and M. Tyunina, Appl. Phys. Lett. 112, 031111 (2018).

${ }^{120}$ M. Lejman, G. Vaudel, I. C. Infante, P. Gemeiner, V. E. Gusev, B. Dkhil, and P. Ruello, Nat. Commun. 5, 4301 (2014).

${ }^{121} \mathrm{H}$. Tompkins and E. Irene, Handbook of Ellipsometry (William Andrew, New York, 2005).

${ }^{122}$ G. E. Jellison, L. A. Boatner, D. H. Lowndes, R. A. McKee, and M. Godbole, Appl. Opt. 33, 6053 (1994).

${ }^{123}$ M. Tyunina, J. Narkilahti, J. Levoska, D. Chvostova, A. Dejneka, V. Trepakov, and V. Zelezny, J. Phys: Condens. Matter 21, 232203 (2009).

${ }^{124}$ A. Dejneka, M. Tyunina, J. Narkilahti, J. Levoska, D. Chvostova, L. Jastrabik, and V. A. Trepakov, Phys. Solid State 52, 2082 (2010).

${ }^{125}$ R. F. Berger, C. J. Fennie, and J. B. Neaton, Phys. Rev. Lett. 107, 146804 (2011).

${ }^{126}$ Y. Tian, C. Adamo, D. G. Schlom, and K. S. Burch, Appl. Phys. Lett. 102, 041906 (2013).

${ }^{127}$ M. Tyunina, L. Yao, D. Chvostova, A. Dejneka, T. Kocourek, M. Jelinek, V. Trepakov, and S. van Dijken, Sci. Technol. Adv. Mater. 16, 026002 (2015).

${ }^{128}$ H. L. Liu, M. K. Lin, Y. R. Cai, C. K. Tung, and Y. H. Chu, Appl. Phys. Lett. 103, 181907 (2013).

${ }^{129}$ G. Catalan, B. Noheda, J. McAneney, L. J. Sinnamon, and J. M. Gregg, Phys. Rev. B 72, 020102(R) (2005).

${ }^{130}$ K. Choi, M. Biegalski, Y. Li, A. Sharan, J. Schubert, R. Uecker, P. Reiche, Y. Chen, X. Pan, V. Gopalan, L.-Q. Chen, D. Schlom, and C. Eom, Science 306, 1005 (2004).

${ }^{131}$ M. Veithen, X. Gonze, and P. Ghosez, Phys. Rev. Lett. 93, 187401 (2004).

${ }^{132}$ M. N. Grisolia, F. Y. Bruno, D. Sando, H. J. Zhao, E. Jacquet, X. M. Chen, L. Bellaiche, A. Barthélémy, and M. Bibes, Appl. Phys. Lett. 105, 172402 (2014).

${ }^{133}$ F. Wang, I. Grinberg, and A. M. Rappe, Appl. Phys. Lett. 104, 152903 (2014).

${ }^{134}$ I. Grinberg, D. V. West, M. Torres, G. Gou, D. M. Stein, L. Wu, G. Chen, E. M. Gallo, A. R. Akbashev, P. K. Davies, J. E. Spanier, and A. M. Rappe, Nature 503, 509 (2013).

${ }^{135}$ X. S. Xu, J. F. Ihlefeld, J. H. Lee, O. K. Ezekoye, E. Vlahos, R. Ramesh, V. Gopalan, X. Q. Pan, D. G. Schlom, and J. L. Musfeldt, Appl. Phys. Lett. 96, 192901 (2010).

${ }^{136}$ A. A. Belik, S. Iikubo, T. Yokosawa, K. Kodama, N. Igawa, S. Shamoto, M. Azuma, M. Takano, K. Kimoto, Y. Matsui, and E. TakayamaMuromachi, J. Am. Chem. Soc. 129, 971 (2007).

${ }^{137}$ O. Diéguez and J. Íñiguez, Phys. Rev. B 91, 184113 (2015).

${ }^{138}$ E. Chernova, O. Pacherova, D. Chvostova, A. Dejneka, T. Kocourek, M. Jelinek, and M. Tyunina, Appl. Phys. Lett. 106, 192903 (2015).

${ }^{139}$ M. J. DiDomenico and S. Wemple, J. Appl. Phys. 40, 720 (1969).

${ }^{140}$ W. Shockley and H. J. Queisser, J. Appl. Phys. 32, 510 (1961).

${ }^{141}$ V. M. Fridkin, Crystallogr. Rep. 46, 654 (2001).

${ }^{142}$ M. Alexe and D. Hesse, Nat. Commun. 2, 256 (2011).

${ }^{143}$ J. E. Spanier, V. M. Fridkin, A. M. Rappe, A. R. Akbashev, A. Polemi, Y. Qi, Z. Gu, S. M. Young, C. J. Hawley, D. Imbrenda, G. Xiao, A. L. Bennett-Jackson, and C. L. Johnson, Nat. Photonics 10, 611 (2016).

${ }^{144}$ A. G. Chynoweth, Phys. Rev. 102, 705 (1956).

${ }^{145}$ A. Bhatnagar, A. R. Chaudhuri, Y. H. Kim, D. Hesse, and M. Alexe, Nat. Commun. 4, 2835 (2013).

${ }^{146}$ J. Seidel, L. W. Martin, Q. He, Q. Zhan, Y.-H. Chu, A. Rother, M. E. Hawkridge, P. Maksymovych, P. Yu, M. Gajek, N. Balke, S. V. Kalinin, S. Gemming, F. Wang, G. Catalan, J. F. Scott, N. A. Spaldin, J. Orenstein, and R. Ramesh, Nat. Mater. 8, 229 (2009).

${ }^{147}$ S. Farokhipoor and B. Noheda, Phys. Rev. Lett. 107, 127601 (2011).

${ }^{148}$ P. Maksymovych, J. Seidel, Y. H. Chu, P. Wu, A. P. Baddorf, L.-Q. Chen, S. V. Kalinin, and R. Ramesh, Nano Lett. 11, 1906 (2011).

${ }^{149}$ J. Guyonnet, I. Gaponenko, S. Gariglio, and P. Paruch, Adv. Mater. 23, 5377 (2011)

${ }^{150}$ L. He and D. Vanderbilt, Phys. Rev. B 68, 134103 (2003).
${ }^{151}$ C. Paillard, G. Geneste, L. Bellaiche, and B. Dkhil, J. Phys: Condens. Matter 29, 485707 (2017).

${ }^{152}$ V. M. Fridkin, E. P. Efremova, B. H. Karimov, V. A. Kuznezov, I. P. Kuzmina, A. N. Lobachev, V. G. Lazarev, and A. J. Rodin, Appl. Phys. 25, 77 (1981)

${ }^{153}$ B. I. Sturman and V. M. Fridkin, The Photovoltaic and Photorefractive Effects in Noncentrosymmetric Materials (Gordon and Breach Science Publishers, 1992).

${ }^{154}$ K. T. Butler, J. M. Frost, and A. Walsh, Energy Environ. Sci. 8, 838 (2015).

${ }^{155}$ A. M. Glass, D. Von Der Linde, and T. J. Negran, Appl. Phys. Lett. 25, 233 (1974).

${ }^{156}$ A. M. Glass, D. von der Linde, D. H. Auston, and T. J. Negran, J. Electron. Mater. 4, 915 (1975).

${ }^{157}$ A. Zenkevich, Y. Matveyev, K. Maksimova, R. Gaynutdinov, A. Tolstikhina, and V. Fridkin, Phys. Rev. B 90, 161409(R) (2014).

${ }^{158}$ W. Ji, K. Yao, and Y. C. Liang, Phys. Rev. B 84, 094115 (2011).

${ }^{159}$ S. M. Young and A. M. Rappe, Phys. Rev. Lett. 109, 116601 (2012).

${ }^{160}$ S. M. Young, F. Zheng, and A. M. Rappe, Phys. Rev. Lett. 109, 236601 (2012).

${ }^{161}$ F. Wang, S. M. Young, F. Zheng, I. Grinberg, and A. M. Rappe, Nat. Commun. 7, 10419 (2016).

${ }^{162}$ T. Qi, I. Grinberg, and A. M. Rappe, Phys. Rev. B 83, 224108 (2011).

${ }^{163}$ R. Nechache, C. Harnagea, S. Licoccia, E. Traversa, A. Ruediger, A. Pignolet, and F. Rosei, Appl. Phys. Lett. 98, 202902 (2011).

${ }^{164}$ D. Lee, S. H. Baek, T. H. Kim, J.-G. Yoon, C. M. Folkman, C. B. Eom, and T. W. Noh, Phys. Rev. B 84, 125305 (2011).

${ }^{165}$ Y. S. Yang, S. J. Lee, S. Yi, B. G. Chae, S. H. Lee, H. J. Joo, and M. S. Jang, Appl. Phys. Lett. 76, 774 (2000).

${ }^{166}$ R. Guo, L. You, L. Chen, D. Wu, and J. Wang, Appl. Phys. Lett. 99, 122902 (2011)

${ }^{167}$ Y. Guo, B. Guo, W. Dong, H. Li, and H. Liu, Nanotechnology 24, 275201 (2013)

${ }^{168}$ R. Guo, L. You, Y. Zhou, Z. S. Lim, X. Zou, L. Chen, R. Ramesh, and J. Wang, Nat. Commun. 4, 1990 (2013).

${ }^{169}$ S. Y. Yang, L. W. Martin, S. J. Byrnes, T. E. Conry, S. R. Basu, D. Paran, L. Reichertz, J. Ihlefeld, C. Adamo, A. Melville, Y.-H. Chu, C.-H. Yang, J. L. Musfeldt, D. G. Schlom, J. W. Ager III, and R. Ramesh, Appl. Phys. Lett. 95, 062909 (2009).

${ }^{170}$ W. Ji, K. Yao, and Y. C. Liang, Adv. Mater. 22, 1763 (2010).

${ }^{171}$ J. Y. Wang, G. Liu, D. Sando, V. Nagarajan, and J. Seidel, Appl. Phys. Lett. 111, 092902 (2017).

${ }^{172}$ B. Kundys, M. Viret, D. Colson, and D. O. Kundys, Nat. Mater. 9, 803 (2010).

${ }^{173}$ B. Kundys, M. Viret, C. Meny, V. Da Costa, D. Colson, and B. Doudin, Phys. Rev. B 85, 092301 (2012).

${ }^{174}$ B. Kundys, Appl. Phys. Rev. 2, 011301 (2015).

${ }^{175}$ C. Paillard, B. Xu, B. Dkhil, G. Geneste, and L. Bellaiche, Phys. Rev. Lett. 116, 247401 (2016).

${ }^{176}$ C. Paillard, S. Prosandeev, and L. Bellaiche, Phys. Rev. B 96, 045205 (2017).

${ }^{177}$ S. M. Young, F. Zheng, and A. M. Rappe, Phys. Rev. Lett. 110, 057201 (2013).

${ }^{178}$ H. Béa, M. Gajek, M. Bibes, and A. Barthélémy, J. Phys: Condens. Matter 20, 434221 (2008).

${ }^{179}$ V. Iurchuk, D. Schick, J. Bran, D. Colson, A. Forget, D. Halley, A. Koc, M. Reinhardt, C. Kwamen, N. A. Morley, M. Bargheer, M. Viret, R. Gumeniuk, G. Schmerber, B. Doudin, and B. Kundys, Phys. Rev. Lett. 117, 107403 (2016).

${ }^{180} \mathrm{~K}$. Atsuki and E. Yamashita, J. Light. Technol. LT-5, 316 (1987).

${ }^{181}$ J. Allibe, K. Bougot-Robin, E. Jacquet, I. C. Infante, S. Fusil, C. Carretéro, J.-L. Reverchon, B. Marcilhac, D. Crete', J.-C. Mage, A. Barthelémy, and M. Bibes, Appl. Phys. Lett. 96, 182902 (2010).

${ }^{182}$ R. Thapliya, Y. Okano, and S. Nakamura, J. Light. Technol. 21, 1820 (2003).

${ }^{183}$ S. Masuda, A. Seki, K. Shiota, H. Hara, and Y. Masuda, J. Appl. Phys. 109, 124108 (2011).

${ }^{184}$ H. Kogelnik and V. Ramaswamy, Appl. Opt. 13, 1857 (1974).

${ }^{185}$ K. S. Kiyotaka Wasa, O. Yamazaki, H. Adachi, and T. Kawaguchi, J. Light. Technol. LT-2, 710 (1984).

${ }^{186}$ D. Reitze, E. Haton, R. Ramesh, S. Etemad, D. Leaird, T. Sands, Z. Karim, and A. J. Tanguay, Appl. Phys. Lett. 63, 596 (1993).

${ }^{187}$ G. H. Jin, Y. K. Zou, V. Fuflyigin, S. W. Liu, Y. L. Lu, and J. Zhao, J. Lightwave Technol. 18, 807 (2000). 
${ }^{188}$ S. Masuda, A. Seki, K. Shiota, Y. Masuda, A. We, and M. Zehnder, J. Lightwave Technol. 29, 209 (2011).

${ }^{189}$ J. P. George, P. F. Smet, J. Botterman, V. Bliznuk, W. Woestenborghs, D. Van Thourhout, K. Neyts, and J. Beeckman, ACS Appl. Mater. Interfaces 7, 13350 (2015).

${ }^{190}$ D. M. Gill, C. W. Conrad, G. Ford, B. W. Wessels, and S. T. Ho, Appl. Phys. Lett. 71, 1783 (1997).

${ }^{191}$ P. Tang, D. J. Towner, T. Hamano, A. L. Meier, and B. W. Wessels, Opt. Express 12, 5962 (2004).

${ }^{192}$ P. Tang, D. J. Towner, A. L. Meier, and B. W. Wessels, IEEE Photonics Technol. Lett. 16, 1837 (2004).

${ }^{193}$ P. Tang, A. L. Meier, D. J. Towner, and B. W. Wessels, Opt. Lett. 30, 254 (2005).

${ }^{194}$ J. Li, Z. Liu, and B. Wessels, in Frontiers in Optics 2011/Laser Science XXVII, OSA Technical Digest Paper FThW4 (Optical Society of America, 2011).

${ }^{195}$ S. Abel, T. Stöferle, C. Marchiori, C. Rossel, M. D. Rossell, R. Erni, D. Caimi, M. Sousa, A. Chelnokov, B. J. Offrein, and J. Fompeyrine, Nat. Commun. 4, 1671 (2013).

${ }^{196}$ R. A. McKee, F. J. Walker, and M. F. Chisholm, Phys. Rev. Lett. 81, 3014 (1998).

${ }^{197}$ S. Abel, T. Stöferle, C. Marchiori, D. Caimi, L. Czornomaz, M. Stuckelberger, M. Sousa, B. J. Offrein, and J. Fompeyrine, J. Lightwave Technol. 34, 1688 (2016).

${ }^{198}$ C. Xiong, W. H. P. Pernice, J. H. Ngai, J. W. Reiner, D. Kumah, F. J. Walker, C. H. Ahn, and H. X. Tang, Nano Lett. 14, 1419 (2014).

${ }^{199}$ D. Sun, J. Zhang, C. Chen, M. Kong, J. Wang, and H. Jiang, J. Lightwave Technol. 33, 1937 (2015).

${ }^{200}$ M. Bibes and A. Barthélémy, Nat. Mater. 7, 425 (2008).

${ }^{201}$ D. Sando, A. Barthélémy, and M. Bibes, J. Phys: Condens. Matter 26, 473201 (2014)

${ }^{202}$ M. Zhu, Z. Du, Q. Liu, B. Chen, S. H. Tsang, and E. H. T. Teo, Appl. Phys. Lett. 108, 233502 (2016).

${ }^{203}$ V. Babicheva, S. Zhukovsky, and A. Lavrinenko, Opt. Express 22, 28890 (2014)

${ }^{204}$ M. Siegert, W. Zander, J. Lisoni, J. Schubert, and C. Buchal, Appl. Phys. A 69, 779 (1999).

${ }^{205}$ T. Konaka, M. Sato, H. Asano, and S. Kubo, J. Supercond. 4, 283 (1991)

${ }^{206}$ R. E. Stephens and I. H. Malitson, J. Res. Natl. Bur. Stand. 49, 249 (1952).

${ }^{207}$ Handbook of Optical Constants, edited by E. D. Palik (Academic, New York, 1985).

${ }^{208}$ E. C. Chernova, C. B. Brooks, D. C. Chvostova, Z. B. Bryknar, A. D. Dejneka, and M. T. Tyunina, Opt. Mater. Express 7, 3844 (2017).

${ }^{209}$ R. D. Shannon and G. R. Rossman, J. Phys. Chem. Solids 52, 1055 (1991).

${ }^{210}$ H. M. Christen, G. E. Jellison, Jr., I. Ohkubo, S. Huang, M. E. Reeves, E. Cicerrella, J. L. Freeouf, Y. Jia, and D. G. Schlom, Appl. Phys. Lett. 88, 262906 (2006).

${ }^{211}$ T. M. Gesing, R. Uecker, and J.-C. Buhl, Z. Krist. 224, 365 (2009).

${ }^{212}$ R. Uecker, B. Velickov, D. Klimm, R. Bertram, M. Bernhagen, M. Rabe, M. Albrecht, R. Fornari, and D. G. Schlom, J. Cryst. Growth 310, 2649 (2008).

${ }^{213}$ M. D. Agrawal and K. V. Rao, J. Phys. C 3, 1120 (1970).

${ }^{214}$ J. Robertson, J. Vac. Sci. Technol. B 18, 1785 (2000).

${ }^{215}$ G. E. Jellison, I. Paulauskas, L. A. Boatner, and D. J. Singh, Phys. Rev. B: Condens. Matter Mater. Phys. 74, 155130 (2006).

${ }^{216}$ D. G. Schlom, C. A. Billman, J. H. Haeni, J. Lettieri, P. H. Tan, R. R. M. Held, S. Völk, and K. J. Hubbard, in Thin Films and Heterostructures for Oxide Electronics (Springer, 2005), pp. 31-78.

${ }^{217}$ T. Heeg, J. Schubert, C. Buchal, E. Cicerrella, J. L. Freeouf, W. Tian, Y. Jia, and D. G. Schlom, Appl. Phys. A 83, 103 (2006).

${ }^{218}$ M. DiDomenico and S. H. Wemple, Phys. Rev. 166, 565 (1968).
${ }^{219}$ K. Van Benthem, C. Elsässer, and R. H. French, J. Appl. Phys. 90, 6156 (2001).

${ }^{220}$ D. J. Tao, H. X. Wu, X. D. Xu, R. S. Yan, F. Y. Liu, A. P. B. Sinha, X. P. Jiang, and H. L. Hu, Opt. Mater. 23, 425 (2003).

${ }^{221}$ A. H. Reshak, M. Piasecki, S. Auluck, I. V. Kityk, R. Khenata, B. Andriyevsky, C. Cobet, N. Esser, A. Majchrowski, M. Swirkowicz, R. Diduszko, and W. Szyrski, J. Phys. Chem. B 113, 15237 (2009).

${ }^{222}$ A. Dabkowski, H. A. Dabkowski, and J. E. Greedan, J. Cryst. Growth 132, 205 (1993).

${ }^{223}$ X. Q. Liu and X. M. Chen, J. Eur. Ceram. Soc. 26, 1969 (2006).

${ }^{224}$ D. E. Aspnes and A. A. Studna, Phys. Rev. B 27, 985 (1983).

${ }^{225}$ K. Seeger, J. Appl. Phys. 63, 5439 (1988).

${ }^{226}$ S.-Y. Cho, I.-T. Kim, and K. S. Hong, J. Mater. Res. 14, 114 (1999).

${ }^{227}$ M. J. Weber, M. Bass, K. Andringa, R. R. Monchamp, and E. Comperchio, Appl. Phys. Lett. 15, 342 (1969).

${ }^{228}$ R. Diehl and G. Brandt, Mater. Res. Bull. 10, 85 (1975).

${ }^{229}$ T. Tomiki, H. Ishikawa, T. Tashiro, M. Katsuren, A. Yonesu, T. Hotta, T. Yabiku, M. Akamine, T. Futemma, T. Nakaoka, and I. Miyazato, J. Phys. Soc. Jpn. 64, 4442 (1995).

${ }^{230}$ D. L. Wood and K. Nassau, Appl. Opt. 21, 2978 (1982).

${ }^{231}$ V. R. Paiverneker, A. N. Petelin, F. J. Crowne, and D. C. Nagle, Phys. Rev. B 40, 8555 (1989).

${ }^{232}$ M. T. Lanagan, J. K. Yamamoto, a Bhalla, and S. G. Sankar, Mater. Lett. 7, 437 (1989).

${ }^{233}$ I. H. Malitson, J. Opt. Soc. Am. 52, 1377 (1962).

${ }^{234}$ V. E. Wood, J. R. Busch, S. D. Ramamurthi, and S. L. Swartz, J. Appl. Phys. 71, 4557 (1992).

${ }^{235}$ C. M. Foster, G.-R. Bai, R. Csencsits, J. Vetrone, R. Jammy, L. A. Wills, E. Carr, and J. Amano, J. Appl. Phys. 81, 2349 (1997).

${ }^{236}$ G. Shirane, R. Newnham, and R. Pepinsky, Phys. Rev. 96, 581 (1954).

${ }^{237}$ N. Umemura, K. Yoshida, and K. Kato, Appl. Opt. 38, 991 (1999).

${ }^{238}$ M. Cardona, Phys. Rev. 140, A651 (1965).

${ }^{239}$ G. H. Kwei, A. C. Lawson, S. J. L. Billinge, and S. W. Cheong, J. Phys. Chem. 97, 2368 (1993).

${ }^{240}$ S. Singh, Appl. Phys. Lett. 20, 135 (1972).

${ }^{241}$ S. A. Mabud and A. M. Glazer, J. Appl. Crystallogr. 12, 49 (1979).

${ }^{242}$ N. Izyumskaya, Y. I. Alivov, S. J. Cho, H. Morkoç, H. Lee, and Y. S. Kang, Crit. Rev. Solid State Mater. Sci. 32, 111 (2007).

${ }^{243}$ J.-P. Rivera and H. Schmid, Ferroelectrics 204, 23 (1997).

${ }^{244}$ A. Kumar, R. C. Rai, N. J. Podraza, S. Denev, M. Ramirez, Y.-H. Chu, L. W. Martin, J. Ihlefeld, T. Heeg, J. Schubert, D. G. Schlom, J. Orenstein, R. Ramesh, R. W. Collins, J. L. Musfeldt, and V. Gopalan, Appl. Phys. Lett. 92, 121915 (2008).

${ }^{245}$ J. Macutkevic, A. Molak, and J. Banys, Ferroelectrics 479, 48 (2015).

${ }^{246}$ M. Tyunina, D. Chvostova, L. D. Yao, A. Dejneka, T. Kocourek, M. Jelinek, and S. Van Dijken, Phys. Rev. B 92, 104101 (2015).

${ }^{247}$ D. Schmidt, L. You, X. Chi, J. Wang, and A. Rusydi, Phys. Rev. B 92, 075310 (2015).

${ }^{248} \mathrm{R}$. Weis and T. Gaylord, Appl. Phys. A 37, 191 (1985).

${ }^{249}$ S. Schwyn, H. W. Lehmann, and R. Widmer, J. Appl. Phys. 72, 1154 (1992).

${ }^{250}$ D. E. Zelmon, D. L. Small, and D. Jundt, J. Opt. Soc. Am. B 14, 3319 (1997).

${ }^{251}$ D. H. Jundt, Opt. Lett. 22, 1553 (1997).

${ }^{252}$ S. Çabuk and A. Mamedov, J. Opt. A 1, 424 (1999).

${ }^{253}$ M. Cuniot-Ponsard, J. M. Desvignes, A. Bellemain, and F. Bridou, J. Appl. Phys. 109, 014107 (2011).

${ }^{254}$ D. Kim, S. Lee, and T. W. Noh, Mater. Sci. Eng. B 56, 251 (1998).

${ }^{255}$ K. J. Kormondy, S. Abel, F. Fallegger, Y. Popoff, P. Ponath, A. B. Posadas, M. Sousa, D. Caimi, H. Siegwart, E. Uccelli, L. Czornomaz, C. Marchiori, J. Fompeyrine, and A. A. Demkov, Microelectron. Eng. 147, 215 (2015).

${ }^{256}$ M. Tyunina, L. D. Yao, D. Chvostova, T. Kocourek, M. Jelinek, A. Dejneka, and S. Van Dijken, New J. Phys. 17, 043048 (2015). 